\title{
EVOLUTION OF THE QUASAR LUMINOSITY FUNCTION OVER $3<z<5$ IN THE COSMOS SURVEY FIELD
}

\author{
D. Masters ${ }^{1,2}$, P. Capak ${ }^{3,4}$, M. Salvato ${ }^{5}$, F. Civano ${ }^{6}$, B. Mobasher ${ }^{1}$, B. Siana ${ }^{1}$, G. Hasinger ${ }^{7}$, C. D. Impey ${ }^{8,9}$,

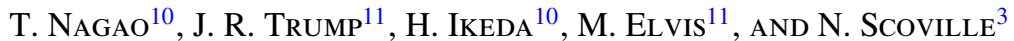 \\ ${ }^{1}$ Department of Physics and Astronomy, University of California, Riverside, CA 92521, USA \\ ${ }^{2}$ Observatories of the Carnegie Institution of Washington, Pasadena, CA 91101, USA \\ ${ }^{3}$ Department of Astronomy, California Institute of Technology, Pasadena, CA 91125, USA \\ ${ }^{4}$ Spitzer Science Center, 314-6 Caltech, Pasadena, CA 91125, USA \\ ${ }^{5}$ Max Planck-Institut für Extraterrestrische Physik, Giessenbachstrasse 1, D-85748 Garching, Germany \\ ${ }^{6}$ Harvard-Smithsonian Center for Astrophysics, 60 Garden Street, Cambridge, MA 02138, USA \\ ${ }^{7}$ Institute for Astronomy, 2680 Woodlawn Drive, University of Hawaii, Honolulu, HI 96822, USA \\ ${ }^{8}$ Department of Astrophysical Science, University of Princeton, Peyton Hall 103, Princeton, NJ 08544, USA \\ ${ }^{9}$ Steward Observatory, University of Arizona, 933 North Cherry Avenue, Tucson, AZ 85721, USA \\ ${ }^{10}$ Research Center for Space and Cosmic Evolution, Ehime University, 2-5 Bunkyo-cho, Matsuyama 790-8577, Japan \\ ${ }^{11}$ University of California Observatories/Lick Observatory and Department of Astronomy and Astrophysics, \\ University of California, Santa Cruz, CA 95064, USA \\ Received 2011 November 29; accepted 2012 July 6; published 2012 August 7
}

\begin{abstract}
We investigate the high-redshift quasar luminosity function (QLF) down to an apparent magnitude of $I_{\mathrm{AB}}=25$ in the Cosmic Evolution Survey (COSMOS). Careful analysis of the extensive COSMOS photometry and imaging data allows us to identify and remove stellar and low-redshift contaminants, enabling a selection that is nearly complete for type-1 quasars at the redshifts of interest. We find 155 likely quasars at $z>3.1,39$ of which have prior spectroscopic confirmation. We present our sample in detail and use these confirmed and likely quasars to compute the rest-frame UV QLF in the redshift bins $3.1<z<3.5$ and $3.5<z<5$. The space density of faint quasars decreases by roughly a factor of four from $z \sim 3.2$ to $z \sim 4$, with faint-end slopes of $\beta \sim-1.7$ at both redshifts. The decline in space density of faint optical quasars at $z>3$ is similar to what has been found for more luminous optical and X-ray quasars. We compare the rest-frame UV luminosity functions found here with the X-ray luminosity function at $z>3$, and find that they evolve similarly between $z \sim 3.2$ and $z \sim 4$; however, the different normalizations imply that roughly $75 \%$ of X-ray bright active galactic nuclei (AGNs) at $z \sim 3-4$ are optically obscured. This fraction is higher than found at lower redshift and may imply that the obscured, type- 2 fraction continues to increase with redshift at least to $z \sim 4$. Finally, the implications of the results derived here for the contribution of quasars to cosmic reionization are discussed.
\end{abstract}

Key words: cosmology: observations - galaxies: luminosity function, mass function - Galaxy: evolution quasars: general

Online-only material: color figures

\section{INTRODUCTION}

The evolution of the quasar luminosity function (QLF) with redshift is a key observational constraint on the growth of supermassive black holes (SMBHs) over cosmic time (Richstone et al. 1998; Kauffmann \& Haehnelt 2000; Wyithe \& Loeb 2003; Marconi et al. 2004). The behavior of the QLF places constraints on the duty cycles of quasars, the growth history of SMBHs, and the coevolution of black holes and their host galaxies (Hopkins et al. 2006; Ueda et al. 2003, and references therein).

The QLF also determines the cumulative ionizing background radiation due to quasars. The faint end of the QLF at high redshift is of particular interest in this regard because faint quasars contribute substantially to the total ionizing background due to quasars. While the peak in quasar activity around $z \sim 2-3$ is responsible for He II reionization at $z \sim 3$ (Reimers et al. 1997; Sokasian et al. 2002), the relative contribution of quasars to hydrogen reionization at $z \sim 6-10$ is not as well constrained. The observed decline in space density of highly luminous quasars at $z>3$ has been taken as evidence that quasars contribute negligibly to hydrogen reionization (Madau et al. 1999; Fan et al. 2006). However, only the most luminous quasars at high redshift can be found in surveys such as Sloan Digital Sky Survey (SDSS), leaving the contribution of faint quasars to the ionizing background unknown.
The faint end is challenging to study at high redshift because of the need for survey fields that are both deep enough to detect faint sources and wide enough to find a statistically significant number of quasars. In addition, follow-up spectroscopy is difficult due to the faintness of the sources. Two groups have recently investigated the faint end of the QLF at $z \sim 4$ : Glikman et al. 2011 (hereafter G11) using parts of the Deep Lens Survey (DLS; Wittman et al. 2002) and NOAO Deep Wide-Field Survey (NDWFS; Jannuzi \& Dey 1999) fields, with a combined sky coverage of $3.76 \mathrm{deg}^{2}$, and Ikeda et al. 2011 (hereafter I11) using the Hubble Space Telescope (HST) Advanced Camera for Surveys (ACS) region of the COSMOS field (Scoville et al. 2007) with a sky coverage of $1.64 \mathrm{deg}^{2}$. The value of the faintend slope $\beta$ measured in $\mathrm{G} 11,-1.6_{-0.6}^{+0.8}$, is consistent with the value of $-1.67_{-0.17}^{+0.11}$ reported in I1 1 for the COSMOS field.

While these studies agree on the faint-end slope of the QLF at $z \sim 4$, they disagree on the absolute space density of lowluminosity quasars by roughly a factor of four, with a higher space density reported in G11. This discrepancy cannot be attributed to cosmic variance (see Section 8.1) and leads to different pictures of quasar evolution. The result reported in G11 implies that the decline in the space density of faint quasars with redshift after the peak at $z \sim 1-2$ eventually stops and possibly reverses, which could make the contribution of quasars to cosmic reionization significant. 


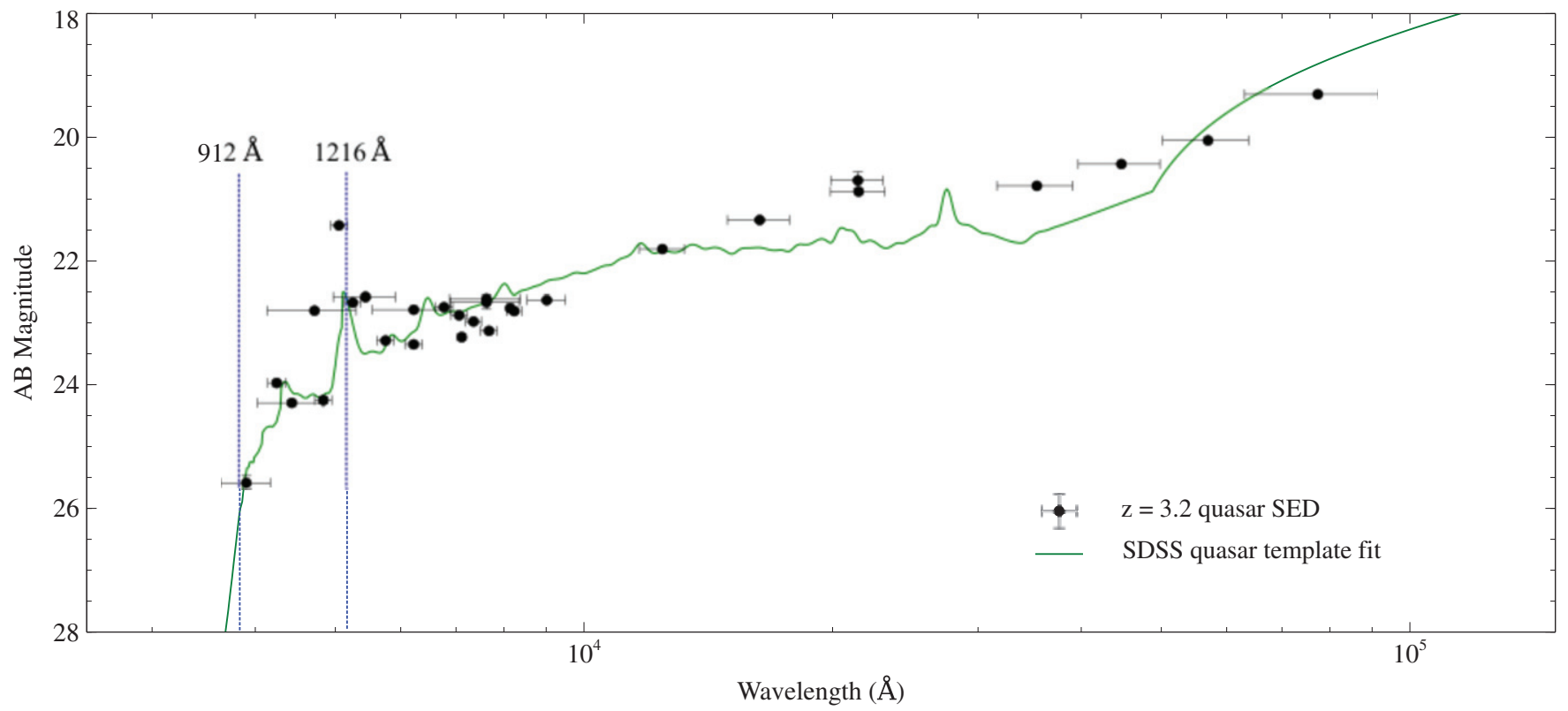

Figure 1. This confirmed quasar at redshift $z=3.2$ illustrates the quality of the low-resolution spectrum afforded by the COSMOS photometric data. Filter bandwidth is indicated with horizontal bars. The observed-frame positions of the Lyman break and Lyman limit are indicated with vertical blue lines. Overlaid is a typical QSO spectral template (Vanden Berk et al. 2001). Features of a high-redshift quasar SED are clear in the photometry, including the Lyman break at rest-frame $1216 \AA$, Ly $\alpha$ emission, broad quasar emission lines, and the rising SED into the observed-frame infrared.

(A color version of this figure is available in the online journal.)

Both I11 and G11 use broadband optical color selection to identify quasars. A significant uncertainty in this approach is the selection function, or the fraction of quasars that are selected as a function of redshift and magnitude. The selection function is usually determined with Monte Carlo simulations, which are sensitive to the assumed distribution of quasar spectral energy distributions (SEDs) in the survey field. This distribution is uncertain, and mismatches between the assumed and actual distributions can give rise to significant errors in completeness estimation and thus the derived luminosity function.

In addition to selection function uncertainty, contamination from stars and galaxies can be substantial and difficult to quantify. Spectroscopy allows a robust determination of the contamination, but due to the large amount of telescope time required, often only a subset of the candidates can be observed. Extrapolating to the complete sample can result in large errors in the estimated contaminant fraction.

Here we take a different approach (described in Section 2), using the COSMOS survey data to select and identify highredshift quasars with high completeness and low contamination. Our principal goals are to (1) determine the faint end of the QLF from $3.1<z<5$ in COSMOS, thereby providing an independent check of the result reported by $\mathrm{I} 11$, as well as a direct determination of the evolution of the faint end over this redshift range; (2) compare the rest-frame UV QLF with the rest-frame $2-10 \mathrm{keV} \mathrm{X-ray} \mathrm{luminosity} \mathrm{function} \mathrm{for} \mathrm{sources} \mathrm{at}$ $z>3$ in order to investigate the obscured fraction of bright active galactic nuclei (AGNs) at these redshifts; and (3) use our results to make inferences regarding the evolution of the QLF and the likely contribution of quasars to reionization, both of He II at $z \sim 3$ and $\mathrm{H}$ I at $z \sim 6-10$.

The structure of this paper is as follows. In Section 2, we provide an overview of the method used to determine the QLF. In Section 3, we present our initial quasar selection. In Section 4, we describe the tests used to verify the high completeness of the selection. In Section 5, we describe how we separate stellar and low $-z$ contaminants from the high-redshift quasar population of interest. In Section 6, we present the final sample of confirmed and likely quasars and discuss the X-ray properties of the sample. In Section 7, we use the final list of likely quasars to compute the luminosity function at $z \sim 3.2$ and $z \sim 4$. In Section 8, we discuss sources of error that may influence our results. In Section 9, we compare our results with the X-ray luminosity function at similar redshifts and discuss the implications for the obscured quasar fraction at these redshifts. In Section 10, we discuss the ionizing background due to quasars. In Section 11, we present our main conclusions.

We assume a cosmology with $\Omega_{\Lambda}=0.7, \Omega_{M}=0.3$, and $H_{0}=70 \mathrm{~km} \mathrm{~s}^{-1} \mathrm{Mpc}^{-1}$. Magnitudes are in the AB system.

\section{OVERVIEW OF METHODOLOGY}

We utilize the COSMOS broad, intermediate, and narrowband photometric catalog (Capak et al. 2007; Sanders et al. 2007) for the selection of quasars and identification of contaminants. With 29 bands of well-matched photometry, these data constitute low-resolution spectra for all objects over the wavelength range $0.1-8 \mu \mathrm{m}$ (Figure 1). This is generally sufficient to distinguish stars from high-redshift quasars. With spectra of sufficient quality for all sources in the field, the population of quasars can be well constrained down to the limiting magnitude. Our approach is similar to that of Wolf et al. (2003), who used the 17 filters ( 5 broad and 12 intermediate band) of the COMBO-17 survey to identify quasars. COSMOS is of similar resolution to the COMBO-17 survey in the optical, but significantly deeper and covering a wider wavelength range.

Because neither the properties of the faint, high-redshift quasar population nor the properties of the contaminating stellar population are very well constrained, we individually inspect the SEDs and imaging data to identify quasars and reject contaminants, as described in Section 5. To ensure that our classifications are unbiased, we also cross-check against automated 
$\chi^{2}$ fitting of model SEDs to the low-resolution spectrophotometry of COSMOS (Section 6.2). The two methods produce similar results, but we argue that the visual classification is more accurate because it allows for a broader range of quasar properties as well as the identification of photometry errors that can influence the $\chi^{2}$ result.

The primary method we use can be summarized as follows.

1. Find a coarse selection (based on point-source morphology, the presence of a Lyman-break, and a power-law infrared slope) that is highly complete for type-1 quasars at $z \sim 3-5$, sacrificing reliability to the extent necessary to achieve high completeness.

2. Assess the 29-band photometric and imaging data for each candidate to remove contaminants.

3. Cross-check the resulting quasar list with known highredshift quasars in COSMOS to confirm our ability to recover quasars with high completeness.

4. Compute the luminosity function with the resulting sample.

Several factors specific to the COSMOS field make this approach reasonable. We list these below.

1. Over 40 confirmed $z>3$ quasars are known in COSMOS, which were selected in different ways (X-ray, infrared, optical). These quasars serve as a guide in developing our selection criteria, and provide an important check on our ability to distinguish quasars from contaminants using photometric and imaging data.

2. A large spectroscopic sample of faint AGNs and galaxy candidates obtained by Keck and VLT can be used to test contamination.

3. High-resolution imaging of the COSMOS field with the HST lets us restrict our sample to true point sources, limiting the contamination from high-redshift star-forming galaxies.

4. Accurate photometric redshifts (Ilbert et al. 2009; Salvato et al. 2009) can be used for sources lacking spectroscopic confirmation.

5. Deep Chandra X-ray imaging of the central $0.9 \mathrm{deg}^{2}$ of the COSMOS field allows another check on the completeness and reliability of our approach, and also permits a comparison of our estimated QLF with the X-ray luminosity function at similar redshifts. We present this analysis in Section 9.

\section{INITIAL CANDIDATE SELECTION}

Spectroscopic campaigns, e.g., Lilly et al. (2007), Trump et al. (2009), Ikeda et al. (2011), and P. Capak et al. (in preparation), have confirmed over 40 quasars at $z>3$ in the COSMOS field. These known quasars were originally selected in different ways (X-ray, infrared, optical) and therefore display a range of SEDs. These confirmed sources are used to guide the development of a coarse initial selection, which is then tested for completeness with simulated quasar photometry, as described in Section 4.

It is worth emphasizing that the selection presented here is intended only to be a weak filter against contamination, in order to reduce the number of candidates to a reasonable number while maintaining high completeness. The careful rejection of contaminants occurs later through inspection of the photometric and imaging data, as described in Section 5.

We begin by selecting point sources in the magnitude range $16 \leqslant I \leqslant 25$, restricting our study to the region of COSMOS covered by ACS imaging (1.64 deg $\mathrm{d}^{2}$ ). Spatially extended sources as defined in the Hubble (ACS catalog of Leauthaud et al.

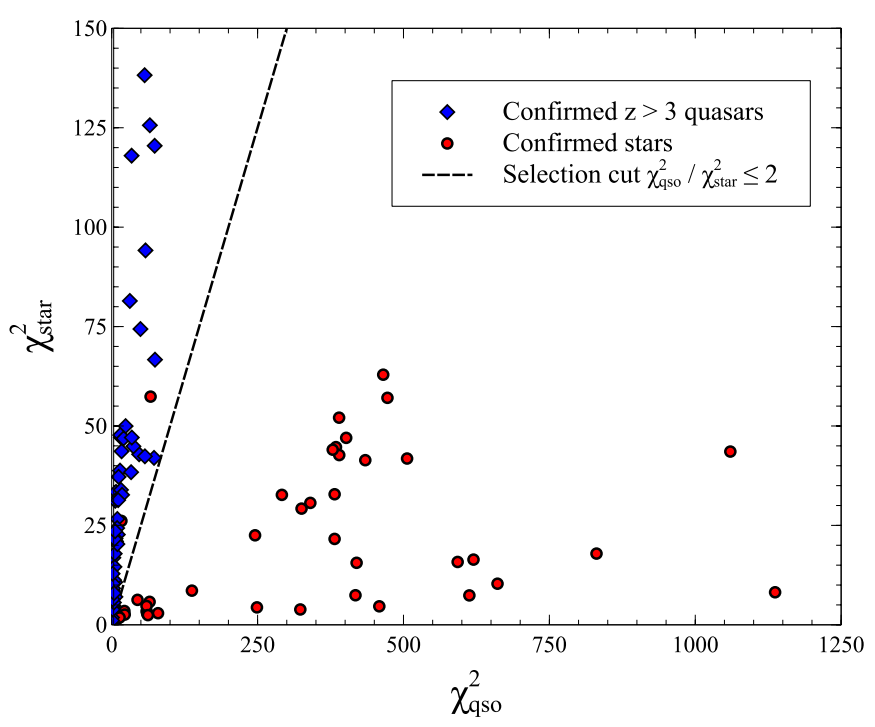

Figure 2. Comparison of the minimum $\chi^{2}$ values for QSO and star template fitting to the COSMOS photometry of point sources with spectra. The cut shown selects objects with $\chi_{\mathrm{qso}}^{2} / \chi_{\text {star }}^{2} \leqslant 2.0$, which retains all known $z>3$ quasars while rejecting a significant fraction of stars. The spectroscopically confirmed stars shown here are primarily F, M, L, and T dwarfs, which are typical contaminants in high-redshift quasar searches (Richards et al. 2009).

(A color version of this figure is available in the online journal.)

(2007) are excluded. The test for point morphology is based on the ratio of the peak surface brightness (MU_MAX) to the magnitude (MAG_AUTO), and exploits the fact that the light distribution of a point source scales with its magnitude. The point-source selection is shown to be robust to $I=25$, which sets the magnitude limit for our search. 22,383 candidates meet this selection criterion.

Next we use the $\chi^{2}$ parameter, computed through template fitting in the COSMOS photometric redshift catalog, to separate stars from quasars. The Le Phare code (Arnouts \& Ilbert 2011) was used to determine photometric redshifts (see Ilbert et al. 2009 for details). As a result of extensive fitting against templates, sources are assigned three $\chi^{2}$ values: $\chi_{\text {gal }}^{2}, \chi_{\mathrm{qso}}^{2}$, and $\chi_{\text {star}}^{2}$, which are computed by fitting galaxy, AGNs, and stellar templates, respectively, to the source photometry. Because we restrict our search to point sources, a comparison between $\chi_{\mathrm{qso}}^{2}$ and $\chi_{\text {star }}^{2}$ can distinguish most stars from quasars. As seen in Figure 2 , the relaxed cut $\chi_{\mathrm{qso}}^{2} / \chi_{\text {star }}^{2} \leqslant 2.0$ selects all known $z>3$ quasars, while rejecting the majority of stars. This leaves us with 8125 candidates.

The effectiveness of $\chi^{2}$ fitting is due to two main factors. One is the COSMOS intermediate-band data, which can differentiate sharp features such as the Lyman break and emission features in quasar photometry from the smooth photometry of stars. The other (more important) factor is Spitzer IRAC photometry. Stars display Rayleigh-Jeans black body curves that decline in the infrared, whereas high-redshift quasars display power-law SEDs that are flat or rising in the infrared.

Next we apply a cut designed to select high-redshift quasars. The Lyman break, caused by the scattering of radiation at wavelengths shorter than $1216 \AA$ by neutral hydrogen in the IGM and quasar host galaxy, results in a reduction in $U$-band flux for quasars at $z \gtrsim 3$.1. In the mid-infrared, however, high-redshift quasars remain relatively bright due to their intrinsic power-law spectral slopes. Therefore, we accept sources meeting either of the following cuts: $U-\operatorname{ch} 1 \geqslant 1.5$ or $U-\operatorname{ch} 2 \geqslant 1.5$. 
Here ch1 and ch2 refer to Spitzer IRAC channel $1(3.6 \mu \mathrm{m})$ and channel $2(4.5 \mu \mathrm{m})$. These cuts were motivated by the spectroscopically confirmed sample as well as simulated quasar spectra and are significantly relaxed in order to achieve high completeness. While the cuts are not significantly different, we accept sources meeting either one in order to avoid losing quasars with bad IRAC photometry in one of the bands. This leaves 6667 candidates. To safeguard against losing unusual quasars, we also accept sources with photometric redshifts $z_{\text {qso }} \geqslant 3$. This adds 28 candidates, for a total of 6695 candidates after this step.

Finally, we require that the source is detected at a significant level $\left(m_{\mathrm{AB}} \leqslant 24.0\right)$ in either IRAC channel 1 or channel 2. Because quasar SEDs typically rise into the infrared, this condition will be met by the majority of quasars with $I<25$, while it serves to eliminate faint stars or other unexpected contaminants. The final number of candidates after applying this selection is 4009 .

This selection, by utilizing only the Lyman continuum break and power-law infrared emission of high-redshift quasars, largely avoids the uncertainties associated with broadband color selection. Color selections in the rest-frame optical must correctly account for intrinsic quasar SEDs that can vary substantially in spectral slope and broad line emission equivalent widths, the distributions of which are not well constrained at high redshift. In addition, this selection should be sensitive to broad absorption line (BAL) quasars, which show similar midinfrared to optical luminosity ratios as non-BALs (Gallagher et al. 2007).

To summarize, the initial (coarse) selection we use is

1. $16<I$ (mag_auto $)<25$;

2. point source based on ACS I-band imaging;

3. $\chi_{\mathrm{qso}}^{2} / \chi_{\mathrm{star}}^{2} \leqslant 2.0$;

4. $U-\operatorname{ch} 1 \geqslant 1.5$ OR $U-\operatorname{ch} 2 \geqslant 1.5$ OR $z_{\text {qso }} \geqslant 3.0$;

5. $m_{\mathrm{AB}}(3.6 \mu \mathrm{m}) \leqslant 24.0 \mathrm{OR} m_{\mathrm{AB}}(4.5 \mu \mathrm{m}) \leqslant 24.0$.

The 4009 candidates selected include all 39 known $z \geqslant 3.1$ quasars in COSMOS.

A comparison of our selection with the high-redshift X-ray sources reported in Civano et al. (2011) confirms that we select all high-redshift X-ray sources with $I \leqslant 25$ and point morphology (27 total). The other 74 sources in the X-ray $z>3$ sample are primarily classified as extended (90\%), or lie outside our I-band magnitude limit, or both. Keck DEIMOS spectra (P. Capak et al., in preparation) of 12 of the extended $z>3$ $\mathrm{X}$-ray sources confirms that these are obscured, type-2 AGNs without broad emission lines, and therefore not appropriate to include in this work.

These pieces of evidence indicate that the selection outlined above is highly complete for unobscured quasars at $z>3.1$. This is verified using simulated quasar photometry, as described in the next section.

\section{SELECTION COMPLETENESS}

We follow the standard procedure of testing the selection by simulating a large number of quasars in redshift/magnitude space. We use the three composite spectral templates QSO1, TQSO1, and BQSO1 from the SWIRE template library (Polletta et al. 2007), each representing a different infrared/optical flux ratio, and extend them into the far-UV using the composite $H S T$ spectral template of Telfer et al. (2002). By letting the intrinsic spectral slopes for $\lambda>1216 \AA$ and $\lambda<1216 \AA$ vary randomly according to the distributions given in Vanden Berk et al. (2001) and Telfer et al. (2002), we generate 50 QSO1 templates and 25 each of the TQSO1 and BQSO1 templates. These 100 base templates are checked against the photometry of known quasars in SDSS, including IRAC photometry for quasars in the Spitzer Heritage Archive, ${ }^{12}$ to verify that they are representative of the underlying variation in the population of type-1 quasars.

We create a grid in redshift and $I$ magnitude, with the redshift extending from $3 \leqslant z \leqslant 5$ in steps of $\Delta z=0.1$ and the $I$-band magnitude extending from $20 \leqslant I \leqslant 26$ in steps of $\Delta I=0.1$. At each point in this grid we generate 20 realizations of each of the 100 model quasar templates, adding Poisson and background noise to the measured flux in each filter, and correcting for intergalactic extinction using the model of Madau (1995). The selection criteria (4) and (5) are then applied to each of the 2000 resulting SEDs to determine the completeness at that grid point.

The result is shown in Figure 3. For $I<23.8$, the completeness is $>90 \%$ over the redshift range $3.1<z<3.3$ and $\sim 100 \%$ at higher redshifts. The marginal incompleteness for $3.1 \lesssim z \lesssim 3.3$ will be improved by the additional inclusion of sources with $z_{\mathrm{qso}}>3$ in criterion (4). There is some incompleteness for $23.8<I<25$ due to criterion (5), which is accounted for when computing the luminosity function.

Figure 3 demonstrates that the photometric criteria (4) and (5) are highly complete, but it does not account for possible incompleteness introduced by criteria (2) and (3). We briefly discuss the effect these may have.

The third selection criterion $\left(\chi_{\mathrm{qso}}^{2} / \chi_{\mathrm{star}}^{2} \leqslant 2.0\right)$ is based on the results of extensive fitting of the COSMOS sources against spectral templates. Checking this criterion with our modeled quasar templates would not be very revealing, as it would constitute checking simulated photometry against simulated photometry. However, the evidence in Figure 2 gives us confidence that this very relaxed criterion introduces negligible incompleteness.

The point-source criterion can potentially induce incompleteness because ACS imaging, with a point-spread function with FWHM of 0.'12 at the detector, resolves all but the most compact of objects. While this is desirable in that it excludes galaxies from consideration, it may also reject intrinsically faint quasars with host galaxy light contributing to the overall flux. We discuss this issue in more detail in Section 8.4.

\section{EXAMINATION OF CANDIDATES AND IDENTIFICATION OF QUASARS}

The selection described above is highly complete but also unreliable, in the sense that a small fraction of the selected objects actually are quasars at the redshifts of interest. However, careful examination of the COSMOS photometric and imaging data for each of the 4009 candidates is relatively fast and allows us to confidently reject typical contaminants such as dwarf stars and lower-redshift quasars.

Visual examination of the data allows more robust classification than a reliance on SED template fitting. The 29 bands of photometry and corresponding imaging data reveal stars with unusual colors that mimic quasar SEDs closely, often because of photometric contamination, as well as true quasars that automated tests fail to find because of contaminated or unusual photometry. In addition, coarse redshift estimates can be made based on the position of $\operatorname{Ly} \alpha$ emission and/or the Lyman break, which can then be compared with the results from automated fitting.

\footnotetext{
12 http://sha.ipac.caltech.edu
} 


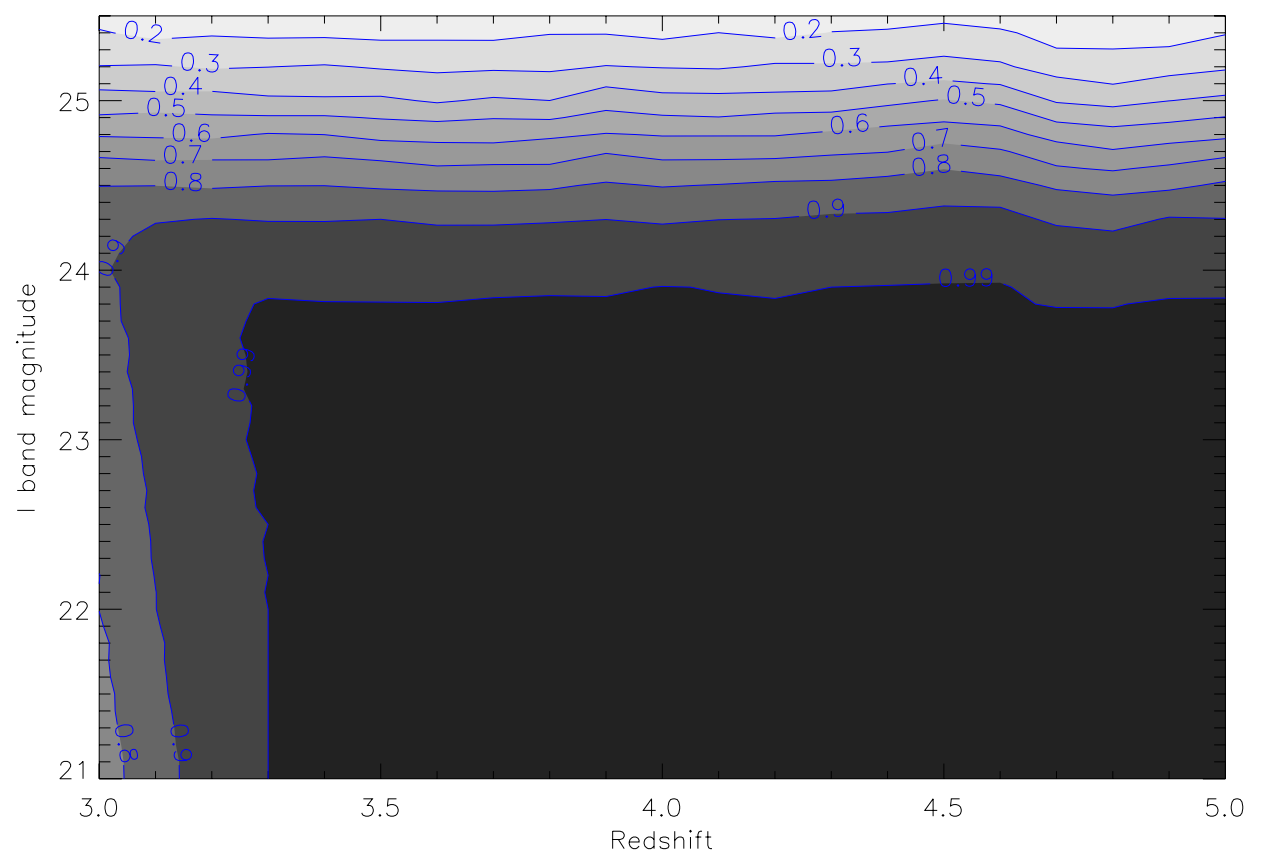

Figure 3. Completeness map for our photometric selection. For magnitudes brighter than $I \sim 24$, the selection is over $90 \%$ complete over the redshift range $3.1 \lesssim z \lesssim 3.3$ and $\sim 100 \%$ complete at higher redshifts. Because we also accept all sources with $z_{\text {qso }} \geqslant 3$, the completeness from $3.1 \lesssim z \lesssim 3.3$ will be enhanced over what is shown. At $I$-band magnitudes fainter than $I \sim 24$ there is some incompleteness due to criterion (5), which is accounted for in computing the luminosity function.

(A color version of this figure is available in the online journal.)

The relevant high-redshift quasar characteristics we look for in the COSMOS photometric and imaging data include (1) a strong spectral break at rest frame $1216 \AA$, (2) a power-law SED that is flat or rising in the infrared IRAC bands, (3) Ly $\alpha$ emission evident in intermediate-band filters, and (4) often other strong rest-frame UV emission lines (e.g., C IV $\lambda 1549$, C III] $\lambda$ 1909) evident in intermediate-band filters as well.

Stars, in contrast, have SEDs with characteristic RayleighJeans shapes that decline in the IRAC bands. The decline in the blue side of a stellar SED is less abrupt than the Lyman break in the SED of a high-redshift quasar, and there are no emission lines in the intermediate bands. Figure 4 illustrates, for some of the most difficult cases, the clear differences in the SEDs of high-redshift quasars and stellar contaminants.

The candidates were examined using Specpro (Masters \& Capak 2011), an interactive IDL tool designed primarily for spectral analysis in the context of multiwavelength surveys. ${ }^{13}$ This program displays the imaging and photometric data for each candidate and allows us to perform rough SED fitting and redshift estimation. The first author went through all candidates after significant calibration against the SEDs of known stars and high-redshift quasars, and subsets of the data were examined by coauthors to establish the consistency and reproducibility of classification. It was found that classifications were highly consistent among different classifiers.

As expected, our sample includes a large fraction of stellar contaminants. Most of these are dwarf stars, while a fraction are stars whose optical fluxes are incorrectly matched to a nearby IR-bright source, which is apparent through examination of the stamp images. The mismatch artificially boosts the SED in the IRAC bands, such that the $\chi_{\mathrm{qso}}^{2}$ versus $\chi_{\mathrm{star}}^{2}$ comparison does not favor a star assignment.

We also find a number of quasars or compact galaxies at $z<3.1$ in our sample, which reflects the fact that our Lyman-

\footnotetext{
13 http://specpro.caltech.edu
}

break criterion (4) is very relaxed. This can also be seen in the slow decline in completeness toward $z=3$ in Figure 3. Lowerredshift objects are identified by the position (or absence) of the Lyman break/Ly $\alpha$ emission in the SED. Of the contaminants, we estimate that $28 \%$ are $z<3.1$ objects (quasars or compact low- $z$ galaxies), and $64 \%$ are stars. The remaining $8 \%$ are unclear based on the photometry but likely fall in one of these categories as they do not show characteristics of $z>3.1$ quasar SEDs.

For the sources identified as likely high-redshift quasars, we assign a confidence flag ranging from 2 to 4 , where 4 is highly confident and 2 is somewhat confident. We emphasize that sources even marginally consistent with being high-redshift quasars are retained (usually at confidence 2) in order to maintain high completeness. Candidates assigned a confidence of 4 typically are X-ray detected and/or show strong emission lines in intermediate bands, as well as a clearly defined Lyman break and rising infrared SED. Candidates assigned a confidence 3 show a well-defined Lyman break and/or Ly $\alpha$ emission evident in an intermediate band filter as well as a rising infrared SED, but are not clearly detected in X-ray. We are confident that these are high-redshift quasars based on their point-source morphology and SED. Candidates assigned a confidence 2 show a moderately convincing photometric break and are flat or rising in the infrared, but do not necessarily show emission features in intermediate bands, nor are they X-ray detected. Of our candidates these are the most likely to be either very compact galaxies or unusual stars rather than quasars. Followup spectroscopy on these sources may be required to refine the results presented here.

\section{QUASAR SAMPLE}

Of 4009 candidates, we find 155 likely type- 1 quasars at $z>3.1$. Of these, 48 are considered confidence 2 sources, 54 are considered confidence 3 , and 53 are considered confidence 4 . 


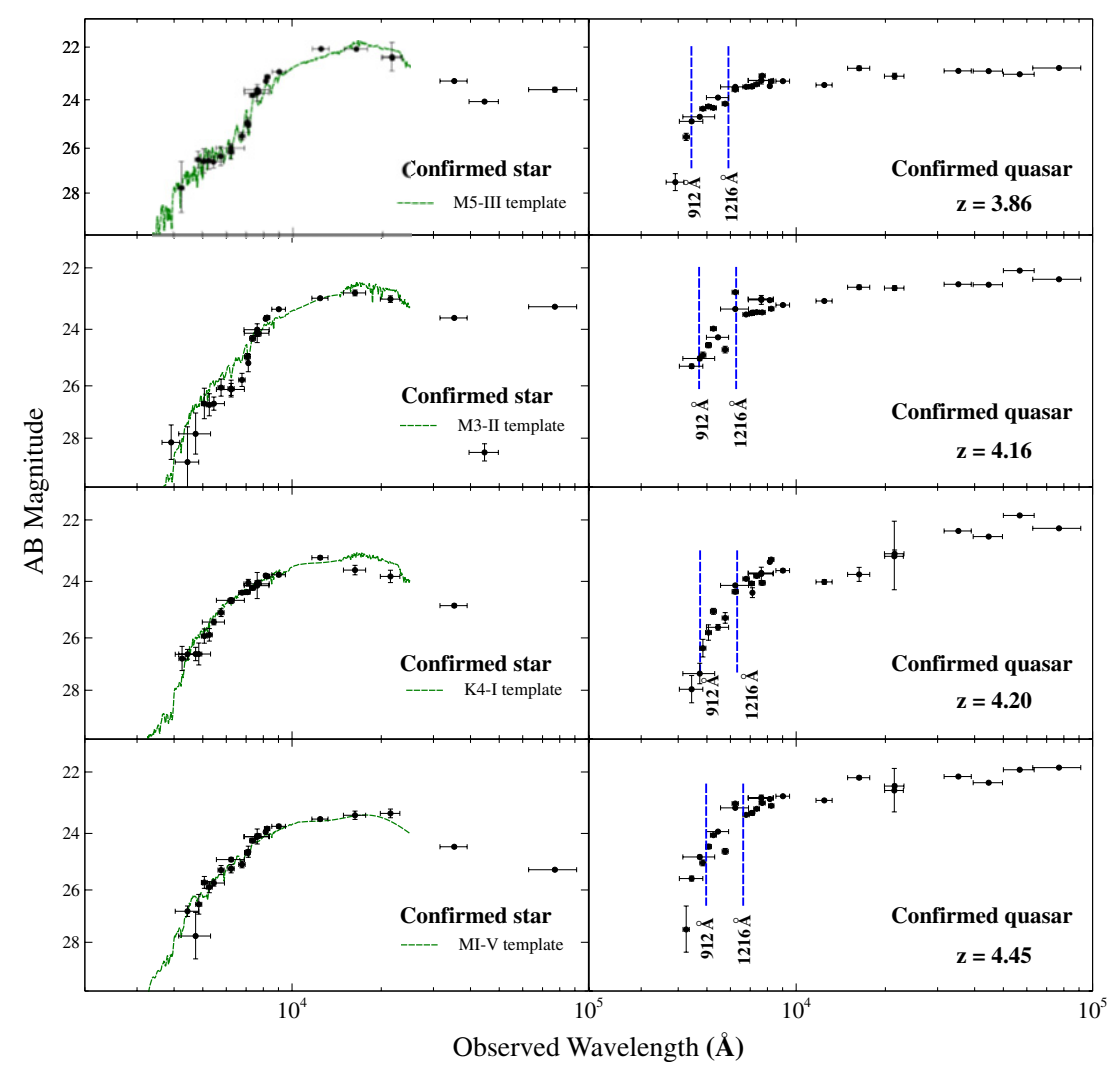

Figure 4. SEDS from the quasar candidates selected in I11. Stars are shown on the left and confirmed quasars on the right. The stars are dwarfs representative of those typically contaminating quasar searches, and the quasars are among the faintest known. Stellar templates (Pickles 1998) are overlaid in green. The obvious differences between the SEDs of the objects make it relatively simple to distinguish them, even without high-resolution spectroscopy. The observed-frame position of the Lyman break and Lyman limit are indicated on the quasar SEDs with vertical blue lines. The distinct break, with a power-law SED in the infrared bands, is characteristic of faint, high-redshift quasars and distinguishes them from stars.

(A color version of this figure is available in the online journal.)

The final list of the 155 confirmed and likely $z>3.1$ quasars is given in Table 3.

\subsection{Identification Reliability}

An important point is that the quasar candidates were examined without knowing which were the previously confirmed quasars, yet everyone of the 39 known quasars at $z \geqslant 3.1$ in the COSMOS field was correctly classified based on the imaging and photometric data. The method employed thus independently recovered every previously known type-1 quasar in the field. Figure 5 shows that the confirmed sample includes a number of very faint sources, including those discovered by Ikeda et al. (2011). This is strong evidence that we do not miss actual high-redshift quasars in the initial sample. If anything, we may overcount by misidentifying either stars or high-redshift galaxies as quasars. Because stars display easily recognizable SEDs, we consider it unlikely that they constitute a significant contaminant.

While stars are relatively easy to reject, high-redshift galaxies display a similar overall SED to a quasar and could contaminate the sample. We expect that few of the candidates identified as quasars are actually compact galaxies, because the strict point-source criterion will eliminate nearly all galaxies at these redshifts. We discuss this potential source of error in more detail in Section 8.3.

\subsection{Comparison with Automated $\chi^{2}$ Selection}

As another check on the reliability of our classifications, we compare our final sample with a purely automated selection

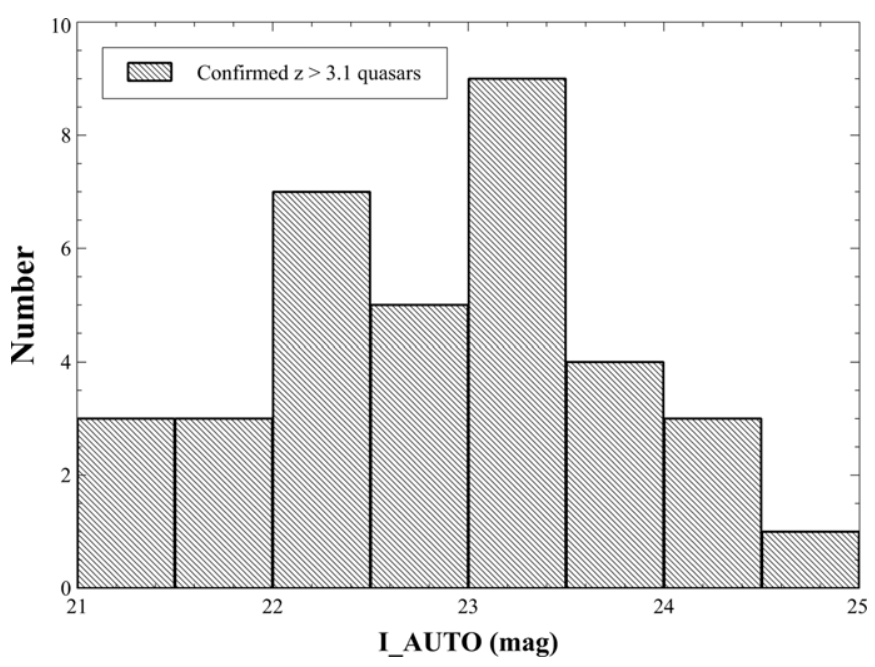

Figure 5. Distribution in $I$ (mag_auto) for the spectroscopically confirmed subset of our final quasar candidates. We have recovered both relatively bright and very faint known quasars in the field, indicating that there is high overall completeness in our classification method regardless of quasar luminosity.

using the results of the extensive $\chi^{2}$ fitting against stellar and AGN templates described in Ilbert et al. (2009). Of the initial sample of 4009 candidates, 195 objects have photometric redshift estimates above $z=3$.1. Limiting the sample to objects with $\chi_{\mathrm{qso}}^{2} / \chi_{\mathrm{star}}^{2} \leqslant 1.0$ (i.e., quasar is the favored solution from $\chi^{2}$ fitting) the final automated sample is 143 objects, in very 


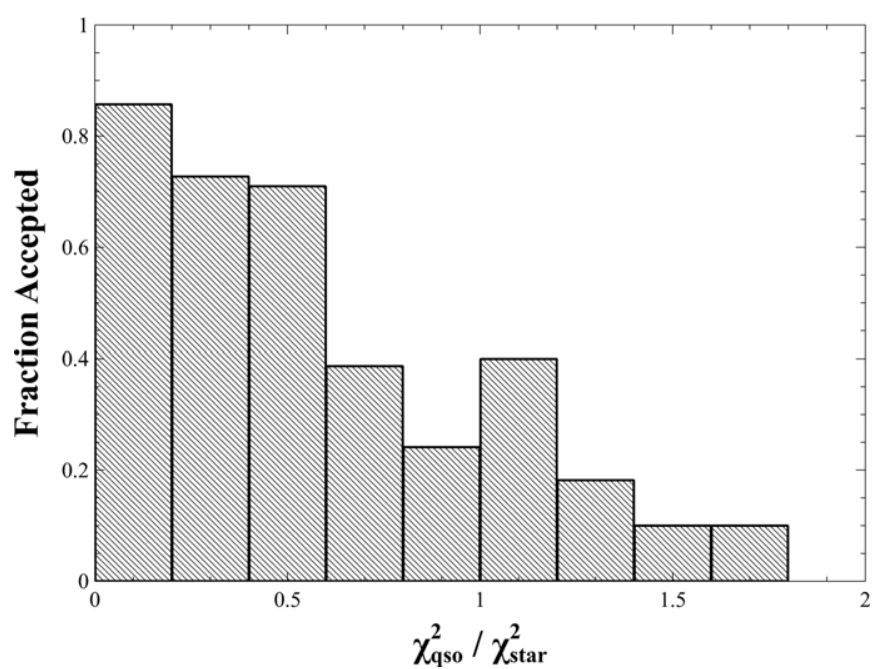

Figure 6. Fraction of the $z_{\text {qso }}>3.1$ candidates we identify as high-redshift quasars as a function of the ratio $\chi_{\mathrm{qso}}^{2} / \chi_{\mathrm{star}}^{2}$. As expected, the lower this ratio, the more likely that we identify a candidate as a quasar through inspection of the imaging and photometry, showing that our visual inspection is in accord with the results of $\chi^{2}$ fitting against spectral templates.

close agreement with our final sample of 155 from visual classification.

Figure 6 shows that, of the 195 objects with photometric redshifts above 3.1 , our visual classification typically retains objects with $\chi_{\text {qso }}^{2} / \chi_{\text {star }}^{2} \lesssim 1$ and rejects objects with $\chi_{\text {qso }}^{2} / \chi_{\text {star }}^{2} \gtrsim$ 1 , as would be expected if the visual classification is in agreement with $\chi^{2}$ fitting.

Of the 143 objects that would be selected automatically, 91 are in common with our final selection. The sources that we rejected but automated fitting would retain are, for the most part, stars mismatched to a nearby IR-bright source, artificially elevating their IRAC fluxes and thus lowering their $\chi_{\mathrm{qso}}^{2}$ value. This is reflected in the fact that these sources have higher average IRAC-to-optical match distances.

On the other hand, we identify 64 likely quasars that are rejected by the automated selection. These primarily are rejected due to a low photometric redshift estimate. However, they are consistent with being high- $z$ quasars based on a weak photometric break and power-law infrared spectrum, so we retain them.

Despite the slightly different samples, it is clear that automated selection based on $\chi^{2}$ fitting produces essentially the same answer as careful rejection of contaminants on an objectby-object basis.

\subsection{X-Ray Properties}

The Chandra COSMOS survey (C-COSMOS; Elvis et al. 2009 ) is a $1.8 \mathrm{Ms}$ Chandra program covering the central $0.5 \mathrm{deg}^{2}$ of the COSMOS field with an effective exposure of $\sim 160 \mathrm{ks}$, and an outer $0.4 \mathrm{deg}^{2}$ area with an effective exposure of $\sim 80 \mathrm{ks}$. In addition, the entire COSMOS field has been observed in the $\mathrm{X}$-ray with XMM-Newton (Hasinger et al. 2007) to a brighter $\mathrm{X}$-ray flux limit.

Of the final sample of confirmed and likely quasars presented here, 27 are X-ray detected by Chandra (Civano et al. 2011, 2012, submitted) and 12 are detected by XMM-Newton (Brusa et al. 2010). The remainder of the objects are undetected and therefore have X-ray fluxes below the detection limits of either Chandra deep, Chandra shallow, or XMM-Newton, depending
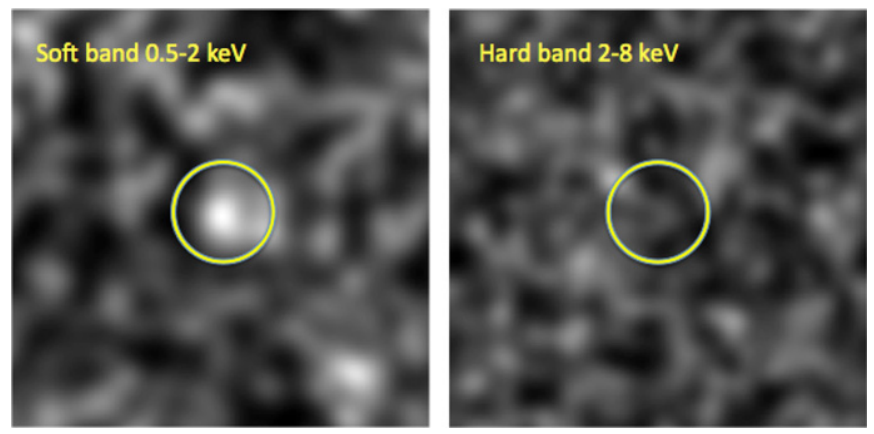

Figure 7. Stacking analysis of 47 likely quasars that fall in the Chandra region but are not individually detected in X-ray. The images are $30^{\prime \prime} \times 30^{\prime \prime}$ and are smoothed with a Gaussian kernel with FWHM of $6^{\prime \prime}$. The $\sim 5 \sigma-6 \sigma$ detection in the soft band (corresponding to the hard X-ray band in the rest frame at $z \sim 3-4)$ is evidence that at least a fraction of the individually undetected sources are quasars. The lack of a clear detection in the observed-frame $2-8 \mathrm{keV}$ stack indicates that they are likely unobscured, intrinsically faint objects that fall below the detection limit in the hard band.

(A color version of this figure is available in the online journal.)

on their location in the COSMOS field. We perform a stacking analysis of 47 unconfirmed sources without X-ray detection in the Chandra region to investigate whether their X-ray fluxes are consistent with expected quasar emission.

We use CSTACK ${ }^{14}$ to compute the number of X-ray counts at the optical position of the undetected sources. This program correctly accounts for the different exposure times depending on the source position. We find a detection in the soft-band $0.5-2.0 \mathrm{keV}$ (Figure 7), corresponding closely to rest-frame $2-10 \mathrm{keV}$ for our sources, with a significance of $\sim 5 \sigma-6 \sigma$, as determined through random stacking in blank fields. We find no clear detection in the observed-frame $2-8 \mathrm{keV}$ hard band. The stacked detection in the soft band provides evidence that these sources are quasars with individual X-ray fluxes below the Chandra detection limit, while the lack of a clear hard-band detection suggests no significant X-ray obscuration.

As another check on whether the X-ray flux limits on the nondetected candidates are consistent with these objects being quasars, we compute the $\alpha_{\text {ox }}$ ratio for sources in the C-COSMOS region, making use of the SEDs to derive the rest-frame UV fluxes. The $\alpha_{\mathrm{ox}}$ parameter represents the spectral slope between the UV and X-ray flux:

$$
\alpha_{o x} \equiv \frac{\log \left(f_{\mathrm{X}} / f_{\mathrm{uv}}\right)}{\log \left(v_{\mathrm{X}} / v_{\mathrm{uv}}\right)},
$$

where $f_{\text {uv }}$ is the $2500 \AA$ flux and $f_{\mathrm{X}}$ is the $2 \mathrm{keV} \mathrm{X}$-ray flux. For X-ray detected sources we derived the $2 \mathrm{keV}$ X-ray flux from the 0.5-2 keV flux (Elvis et al. 2009). For sources without $\mathrm{X}$-ray detection, we compute an upper limit on the X-ray flux, taking into account the X-ray flux limit at the optical position of each source. The result in Figure 8 shows that the nondetected sources tend to have lower UV luminosity, with upper limits on the $\alpha_{\mathrm{ox}}$ ratio that are generally consistent with previously measured correlations between $\alpha_{\mathrm{ox}}$ and the $2500 \AA$ luminosity (Steffen et al. 2006; Young et al. 2010). In conjunction with the stacking results, this gives additional evidence that the sources are faint, type-1 quasars with X-ray fluxes below the Chandra detection limit.

\footnotetext{
14 http://cstack.ucsd.edu/cstack
} 


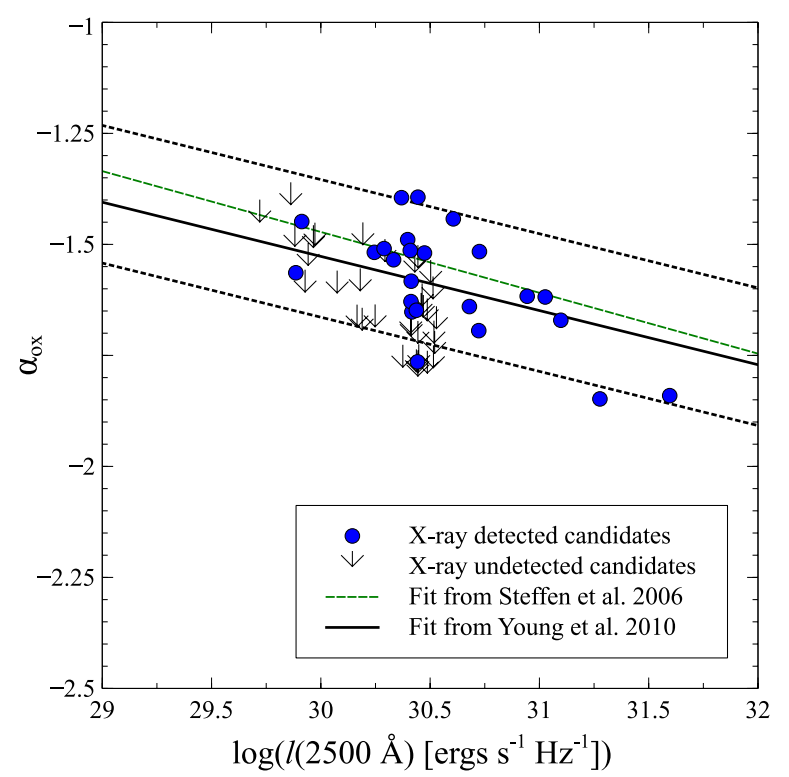

Figure 8. X-ray/UV correlation for the sources in our sample in the Chandra region of COSMOS. The $\alpha_{\mathrm{ox}}$ ratios (or upper limits on the ratio for sources without individual X-ray detection) are generally consistent with measured correlations at lower redshifts. The dotted lines indicate the $1 \sigma$ dispersion in the $\alpha_{o x}$ relation of Young et al. (2010).

(A color version of this figure is available in the online journal.)

\section{LUMINOSITY FUNCTION}

We derive the luminosity function for the redshift bins $3.1 \leqslant z \leqslant 3.5$ and $3.5<z \leqslant 5.0$, using the standard $1 / V_{\max }$ estimator (Schmidt 1968).

The luminosity function for quasars is generally found to be well described by a double power law of the form

$$
\Phi(M, z)=\frac{\Phi\left(M^{*}\right)}{10^{0.4(\alpha+1)\left(M-M^{*}\right)}+10^{0.4(\beta+1)\left(M-M^{*}\right)}},
$$

where $\Phi\left(M^{*}\right)$ is the normalization, $M_{1450}^{*}$ is the break luminosity between the bright and faint ends, $\alpha$ is the bright-end slope, and $\beta$ is the faint-end slope (Boyle et al. 1988). We use this parameterization to fit our results in conjunction with brightend results of Richards et al. (2006) derived in the SDSS. Before presenting the results we first outline the determination of redshift and absolute magnitude for the sample.

\subsection{Redshift Determination}

Only 39 of the 155 sources in the final sample have spectroscopic redshifts. For those that do not, we use the photometric redshift $z_{\text {qso }}$ determined by fitting AGN templates to the source SED (Ilbert et al. 2009). In the cases in which a candidate is $\mathrm{X}$-ray detected but not spectroscopically confirmed, we adopt the photometric redshifts of Salvato et al. (2009, 2011), which have been computed for X-ray sources in COSMOS and are optimized for AGNs.

We check to verify that the photometric redshift is in rough agreement with the redshift estimated from visual examination of the source SED. If there is a very large disagreement between the photometric and visual redshift estimates, we adopt the visually estimated redshift. This accounts for occasional failures of the photometric redshift determination due to contaminated photometry in a small number of bands.

In Figure 9, we show both the photometric redshift and estimated redshift compared with the spectroscopic redshift

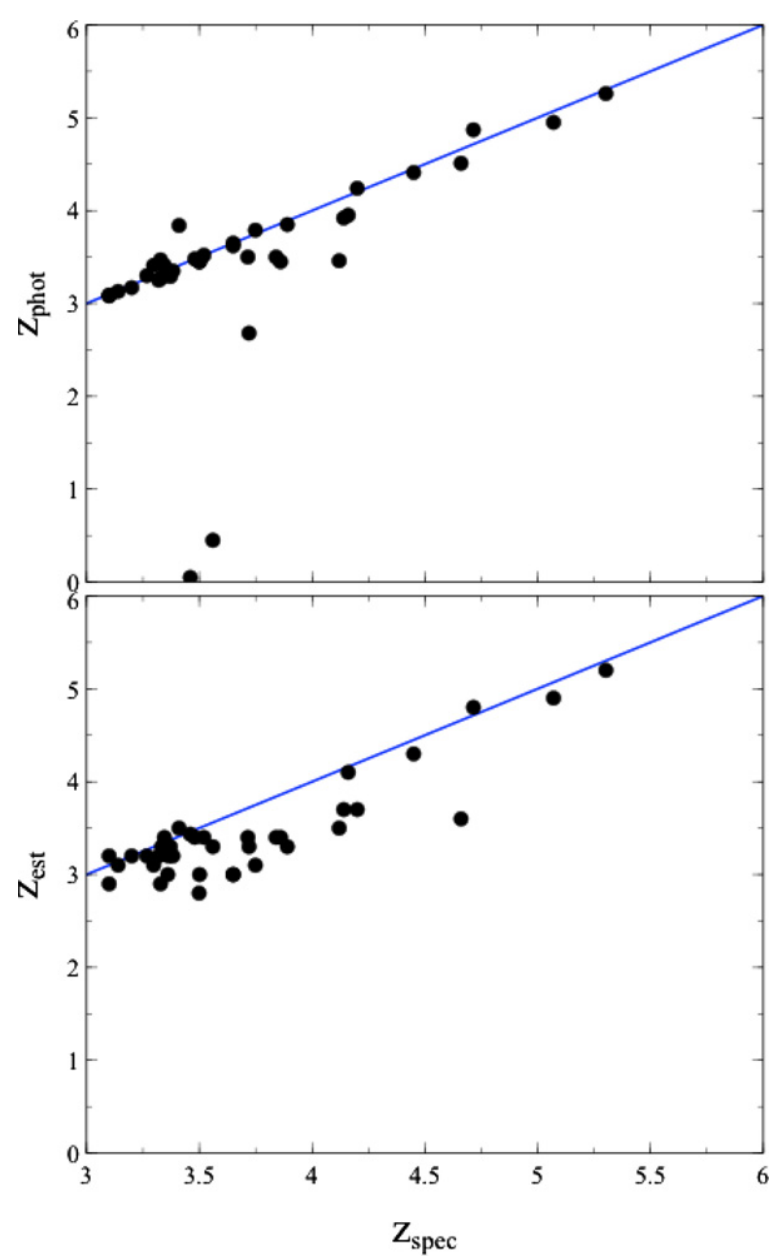

Figure 9. Top: the photometric vs. spectroscopic redshift for the sources in our sample with spectroscopic confirmation. The scatter is $\sim 0.7$, with a few outliers. Bottom: the estimated redshifts vs. spectroscopic redshifts for the same sources. Our visual estimates are systematically low, but with no outliers. These values are only adopted when no spectroscopic redshift is available and the photometric redshift is clearly wrong. This amounts to 29 out of 80 sources in the $3.1<z<3.5$ bin and 9 out of 46 sources in the $3.5<z<5$ bin.

(A color version of this figure is available in the online journal.)

for the subset of spectroscopically confirmed candidates. The photometric redshift is generally more accurate, but contains a small number of outliers. The visually estimated redshifts are low on average, but there are no outliers. These estimated redshifts were made by identifying the position of the Lymanbreak/Lyman-limit in the source SED. The fact that they are systematically low by a small amount reflects a small bias in this visual estimate. We only adopt these values in cases in which the photometric redshift is likely incorrect, primarily as a result of contaminated photometry ( 29 of the 80 sources in the $3.1<z<3.5$ bin and 9 of the 48 sources in the $3.5<z<5$ bin). Because our redshift bins are relatively large, the errors introduced by doing so will not have a significant effect on the derived luminosity functions.

\subsection{Absolute Magnitude Determination}

We follow the convention of giving the QLF in terms of the absolute magnitude at rest-frame $1450 \AA$. To estimate $M_{1450}$, we fit quasar templates to the source SED, which accounts for elevated fluxes due to quasar emission lines. We found that fitting to the measured magnitudes in the three broadband Subaru filters $V, R$, and $I$ and interpolating to find the observed-frame 


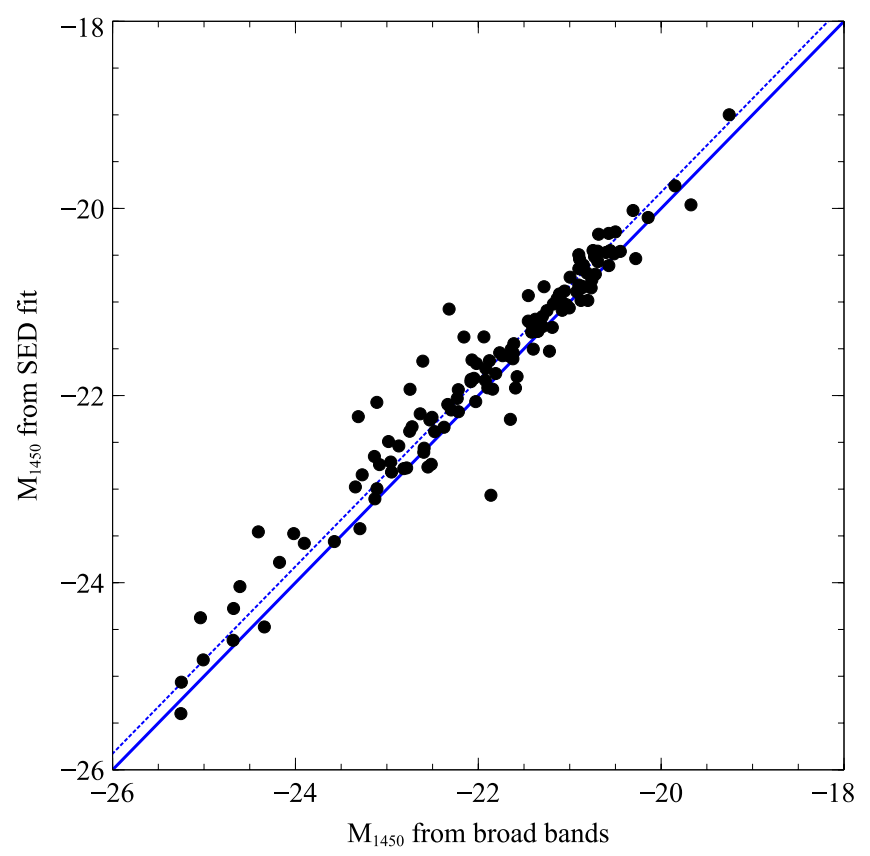

Figure 10. Comparison of the $M_{1450}$ value derived from a linear fit to broadband filters vs. that derived from SED fitting. The absolute magnitude estimated using the SED fit is fainter by $\sim 0.2 \mathrm{mag}$ on average, as indicated with the dotted line. This reflects the fact that the broadband fluxes can be elevated relative to the underlying continuum by broad emission lines.

(A color version of this figure is available in the online journal.)

$1450 \AA$ magnitude often overestimates the luminosity due to the contribution of quasar emission lines to the measured broadband magnitudes.

When performing the SED fits to find $M_{1450}$, only filters within $\pm 2000 \AA$ of the observed-frame wavelength corresponding to rest-frame $1450 \AA$ are considered. The $M_{1450}$ values we find through SED fitting are typically $\sim 0.2$ mag fainter than what we found by extrapolating the broadband magnitudes, but with significant scatter (Figure 10).

Given the observed magnitude $m$ corresponding to rest-frame $1450 \AA$ radiation, the absolute magnitude is found with

$$
M_{1450}=m-5 \log \left(d_{L} / 10\right)+2.5 \log (1+z) .
$$

The second term on the right-hand side is the distance modulus; the final term is a correction for the effective narrowing of the frequency interval sampled in the observed frame.

\subsection{The Luminosity Function at $z \sim 3.2$ and $z \sim 4$}

We compute the luminosity function using the derived redshifts and absolute magnitudes for the sample, correcting for the incompleteness at $I \gtrsim 24$ from the selection function in Figure 3. The results are summarized in Tables 1 and 2. The luminosity function we derive for the redshift bin $3.1<z<3.5$ is shown in Figure 11. There is good continuity with the brightend result derived in the SDSS, as well as close agreement with prior results that have probed the faint end at similar redshift (e.g., Siana et al. 2008; Bongiorno et al. 2007; Wolf et al. 2003).

We fit the $z \sim 3.2$ QLF to the double power-law relationship (Equation (2)) using our faint-end result in conjunction with the bright end from Richards et al. (2006). The best-fit parameters we derive are $\Phi^{*}=2.65( \pm 2.22) \times 10^{-7}, M_{1450}^{*}=-25.54 \pm$ $0.68, \alpha=-2.98 \pm 0.21$, and $\beta=-1.73 \pm 0.11$.

In Figure 12, we show the result for the $z \sim 4$ luminosity function, together with the previous results from G11 and I11.

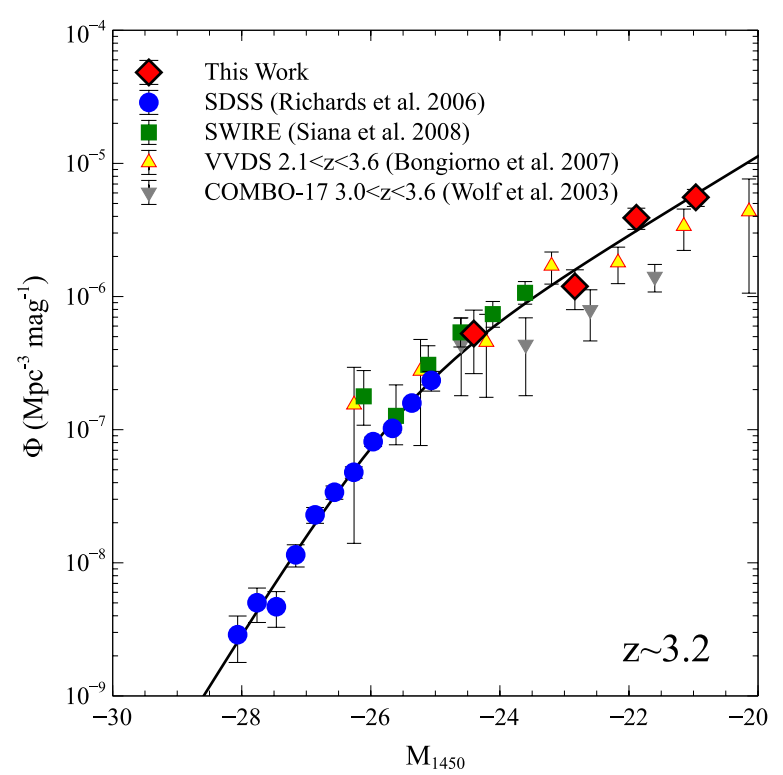

Figure 11. $z \sim 3.2$ luminosity function derived here compared with previous results. The solid black line is our best fit to the double power-law parameterization including the bright-end result from the SDSS. Our result is in close agreement with previous determinations of the QLF at similar redshift. The faint-end slope we derive is $\beta=-1.73 \pm 0.11$.

(A color version of this figure is available in the online journal.)

Table 1

Binned Luminosity Function at $z \sim 3.2$ and $z \sim 4$

\begin{tabular}{llcc}
\hline \hline$\Delta M_{1450}$ & $N_{\mathrm{QSO}}$ & $\begin{array}{c}\Phi\left(\times 10^{-7}\right) \\
\left(\mathrm{Mpc}^{-3} \mathrm{mag}^{-1}\right)\end{array}$ & $\Delta \Phi\left(\times 10^{-7}\right)$ \\
\hline
\end{tabular}

$z \sim 3.2$

\begin{tabular}{|c|c|c|}
\hline$[-25.5,-23.5]$ & 4 & 5.3 \\
\hline
\end{tabular}

$\left[\begin{array}{llll}{[-23.5,-22.5]} & 9 & 11.9 & 3.9\end{array}\right.$

$\left[\begin{array}{llll}-22.5,-21.5] & 26 & 39.0 & 7.1\end{array}\right.$

$\left[\begin{array}{llll}{[-21.5,-20.5]} & 41 & 59.8 & 8.4\end{array}\right.$

$z \sim 4$

$\left[\begin{array}{llll}{[-25.5,-23.5]} & 5 & 1.9 & 0.8\end{array}\right.$

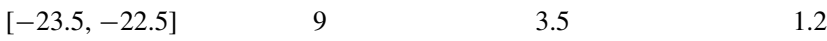

\begin{tabular}{|c|c|}
\hline$[-22.5,-21.5]$ & 14 \\
\hline
\end{tabular}

$\left[\begin{array}{llll}{[-21.5,-20.5]} & 20 & 20.6 & 2.6\end{array}\right.$

Table 2

Double Power-law Parameters, Combining SDSS and this Work

\begin{tabular}{lccc}
\hline \hline$\Phi\left(M^{*}\right)$ & $M_{1450}^{*}$ & $\alpha$ & $\beta$ \\
\hline$z \sim 3.2$ & & & \\
$2.65( \pm 2.22) \times 10^{-7}$ & $-25.54 \pm 0.68$ & $-2.98 \pm 0.21$ & $-1.73 \pm 0.11$ \\
$z \sim 4$ & & & \\
$7.5( \pm 23.5) \times 10^{-8}$ & $-25.64 \pm 2.99$ & $-2.60 \pm 0.63$ & $-1.72 \pm 0.28$ \\
\hline
\end{tabular}

The best-fit parameters to the double power-law parameterization are $\Phi^{*}=7.5( \pm 23.5) \times 10^{-8}, M_{1450}^{*}=-25.64 \pm 2.99$, $\alpha=-2.60 \pm 0.63$, and $\beta=-1.72 \pm 0.28$. Our faint-end result is in agreement with the result reported by I11, and the faint-end slopes we find at both $z \sim 3.2$ and $z \sim 4$ agree with the faintend slope at $2<z<3.6(\beta=-1.70)$ derived in Jiang et al. (2006). However, the space density of faint quasars at $z \sim 4$ we find is lower than the result reported in G11 by a factor of $\sim 3-4$. The source of this discrepancy is unclear, but may be due to contamination (dwarf stars and high-redshift galaxies) in the sample of G11 at the faintest magnitudes probed in that work. The point-source selection used in G11 is done with 
Table 3

Summary of the Likely Quasars Found Here and Used to Compute the Luminosity Function

\begin{tabular}{|c|c|c|c|c|c|c|c|c|c|c|}
\hline ID & $\begin{array}{l}\text { R.A. } \\
\text { (J2000) }\end{array}$ & $\begin{array}{c}\text { Decl. } \\
\text { (J2000) }\end{array}$ & $\begin{array}{c}I_{\mathrm{AB}} \\
(\mathrm{AUTO})\end{array}$ & $z_{\text {est }}$ & $z_{\text {qso }}$ & $z_{\text {spec }}$ & $z_{\text {used }}$ & Flag & X-Ray & $M_{1450}$ \\
\hline 381470 & 149.85396 & 1.753672 & 22.55 & 3.30 & 0.11 & -99 & 3.30 & 4 & no & -22.11 \\
\hline 1625825 & 150.43993 & 2.703496 & 23.05 & 3.43 & 0.05 & 3.46 & 3.46 & 4 & yes & -21.99 \\
\hline 1199385 & 150.39026 & 2.445338 & 24.07 & 3.18 & 2.78 & -99 & 3.18 & 4 & no & -20.60 \\
\hline 1575750 & 150.73715 & 2.722578 & 22.23 & 3.20 & 3.25 & 3.32 & 3.32 & 4 & yes & -22.78 \\
\hline 910592 & 150.73557 & 2.199578 & 20.22 & 3.00 & 3.45 & 3.50 & 3.50 & 4 & yes & -25.06 \\
\hline 1163086 & 150.70377 & 2.370019 & 22.44 & 3.10 & 3.79 & 3.75 & 3.75 & 4 & yes & -23.00 \\
\hline 1159815 & 150.63844 & 2.391350 & 22.16 & 3.00 & 3.62 & 3.65 & 3.65 & 4 & yes & -22.98 \\
\hline 1605275 & 150.62006 & 2.671402 & 21.83 & 3.10 & 3.13 & 3.14 & 3.14 & 4 & yes & -22.82 \\
\hline 887716 & 149.49590 & 1.968019 & 22.61 & 3.30 & 3.23 & -99 & 3.23 & 4 & no & -22.38 \\
\hline 1381605 & 150.57439 & 2.552482 & 23.60 & 3.20 & 3.13 & -99 & 3.13 & 4 & no & -21.54 \\
\hline 503666 & 150.55476 & 1.904693 & 23.90 & 3.00 & 3.33 & -99 & 3.33 & 4 & no & -21.22 \\
\hline 299482 & 150.49628 & 1.638000 & 23.99 & 3.30 & 3.35 & -99 & 3.35 & 4 & no & -21.31 \\
\hline 1856470 & 150.47568 & 2.798362 & 21.28 & 3.50 & 3.46 & 4.12 & 4.12 & 4 & no & -24.37 \\
\hline 507779 & 150.48563 & 1.871927 & 22.03 & 4.30 & 4.41 & 4.45 & 4.45 & 4 & no & -23.78 \\
\hline 739700 & 150.45497 & 1.967424 & 24.03 & 3.40 & 3.48 & 3.48 & 3.48 & 4 & yes & -21.20 \\
\hline 504209 & 150.42476 & 1.900266 & 24.79 & 3.40 & 1.18 & -99 & 3.40 & 4 & yes & -20.10 \\
\hline 748601 & 150.38388 & 2.074549 & 23.46 & 3.50 & 3.45 & -99 & 3.45 & 4 & yes & -21.76 \\
\hline 1208399 & 150.25954 & 2.376141 & 21.42 & 3.30 & 2.68 & 3.72 & 3.72 & 4 & yes & -24.47 \\
\hline 1657280 & 150.24078 & 2.659058 & 22.33 & 3.00 & 3.33 & 3.36 & 3.36 & 4 & yes & -22.74 \\
\hline 1448618 & 150.21497 & 2.582674 & 24.52 & 5.20 & 5.26 & 5.30 & 5.30 & 4 & yes & -22.73 \\
\hline 1463661 & 150.20885 & 2.481935 & 20.11 & 3.30 & 3.28 & 3.33 & 3.33 & 4 & yes & -25.40 \\
\hline 1224733 & 150.20898 & 2.438466 & 21.15 & 3.40 & 3.50 & 3.71 & 3.71 & 4 & yes & -24.28 \\
\hline 560579 & 150.21025 & 1.853839 & 22.12 & 3.40 & 3.45 & -99 & 3.45 & 4 & yes & -23.10 \\
\hline 1231613 & 150.21075 & 2.391473 & 22.55 & 2.90 & 3.08 & 3.10 & 3.10 & 4 & yes & -22.33 \\
\hline 565710 & 150.21199 & 1.818717 & 24.08 & 3.50 & 3.48 & -99 & 3.48 & 4 & no & -21.18 \\
\hline 348143 & 150.17534 & 1.649839 & 24.19 & 3.30 & 3.28 & -99 & 3.28 & 4 & no & -20.65 \\
\hline 1900976 & 150.15880 & 2.808512 & 23.06 & 3.50 & 0.83 & -99 & 3.50 & 4 & no & -21.58 \\
\hline 1465836 & 150.13036 & 2.466012 & 23.04 & 3.40 & 3.45 & 3.86 & 3.86 & 4 & no & -22.56 \\
\hline 1221992 & 150.13364 & 2.457429 & 24.14 & 3.10 & 3.13 & -99 & 3.13 & 4 & yes & -20.74 \\
\hline 1226535 & 150.10098 & 2.419435 & 22.33 & 3.60 & 4.51 & 4.66 & 4.66 & 4 & yes & -23.46 \\
\hline 330806 & 150.10738 & 1.759201 & 22.55 & 3.70 & 3.92 & 4.14 & 4.14 & 4 & yes & -22.85 \\
\hline 780598 & 150.09685 & 2.021495 & 24.33 & 3.50 & 3.23 & -99 & 3.23 & 4 & yes & -20.49 \\
\hline 582598 & 150.04268 & 1.872161 & 23.82 & 3.30 & 3.33 & 3.36 & 3.36 & 4 & yes & -21.80 \\
\hline 585189 & 150.00940 & 1.852637 & 23.67 & 3.00 & 3.33 & -99 & 3.33 & 4 & yes & -21.92 \\
\hline 804307 & 150.00438 & 2.038898 & 21.86 & 2.80 & 3.45 & 3.50 & 3.50 & 4 & yes & -23.56 \\
\hline 1261211 & 149.90547 & 2.354014 & 23.19 & 3.20 & 3.30 & 3.27 & 3.27 & 4 & no & -21.92 \\
\hline 1054048 & 149.87920 & 2.225839 & 22.70 & 3.00 & 3.65 & 3.65 & 3.65 & 4 & yes & -22.77 \\
\hline 1249763 & 149.89417 & 2.432972 & 23.29 & 3.20 & 3.35 & 3.38 & 3.38 & 4 & yes & -21.84 \\
\hline 1271385 & 149.86966 & 2.294046 & 21.67 & 3.40 & 3.41 & 3.35 & 3.35 & 4 & yes & -23.42 \\
\hline 1046585 & 149.85153 & 2.276400 & 23.02 & 3.30 & 3.29 & 3.37 & 3.37 & 4 & yes & -22.39 \\
\hline 1511846 & 149.84576 & 2.481679 & 23.11 & 3.20 & 3.33 & 3.36 & 3.36 & 4 & yes & -21.94 \\
\hline 1513806 & 149.78207 & 2.471342 & 23.70 & 3.40 & 3.26 & -99 & 3.26 & 4 & yes & -20.88 \\
\hline 1719143 & 149.75539 & 2.738555 & 22.87 & 3.40 & 3.52 & 3.52 & 3.52 & 4 & yes & -22.26 \\
\hline 1272246 & 149.78381 & 2.452135 & 23.70 & 4.90 & 4.95 & 5.07 & 5.07 & 4 & yes & -22.23 \\
\hline 1273346 & 149.77692 & 2.444306 & 22.78 & 4.10 & 3.95 & 4.16 & 4.16 & 4 & yes & -22.65 \\
\hline 1284334 & 149.77104 & 2.365819 & 24.64 & 3.60 & 3.45 & -99 & 3.45 & 4 & yes & -20.25 \\
\hline 1060679 & 149.73622 & 2.179933 & 23.45 & 3.70 & 4.24 & 4.20 & 4.20 & 4 & yes & -22.23 \\
\hline 1720201 & 149.74873 & 2.732016 & 23.95 & 3.10 & 3.15 & -99 & 3.15 & 4 & yes & -20.54 \\
\hline 422327 & 149.70151 & 1.638375 & 22.41 & 3.20 & 3.17 & 3.20 & 3.20 & 4 & no & -22.49 \\
\hline 1743444 & 149.66605 & 2.740230 & 22.51 & 3.20 & 3.15 & -99 & 3.15 & 4 & no & -22.54 \\
\hline 1551171 & 149.52461 & 2.531040 & 22.74 & 3.30 & 3.27 & -99 & 3.27 & 4 & no & -21.71 \\
\hline 1330271 & 149.52908 & 2.380164 & 20.80 & 3.20 & 3.09 & 3.10 & 3.10 & 4 & yes & -24.04 \\
\hline 699705 & 150.58488 & 2.081361 & 23.12 & 3.00 & 3.29 & -99 & 3.29 & 4 & yes & -21.83 \\
\hline 1371806 & 150.59184 & 2.619375 & 22.83 & 3.00 & 3.12 & -99 & 3.12 & 3 & no & -22.10 \\
\hline 1628943 & 150.55078 & 2.682909 & 23.91 & 3.30 & 0.45 & 3.56 & 3.56 & 3 & no & -21.31 \\
\hline 361333 & 149.92613 & 1.724807 & 23.46 & 3.10 & 3.41 & 3.30 & 3.30 & 3 & no & -21.57 \\
\hline 1518518 & 149.69594 & 2.603082 & 24.24 & 3.30 & 3.29 & -99 & 3.29 & 3 & no & -20.64 \\
\hline 388046 & 149.72974 & 1.704067 & 23.62 & 3.30 & 0.17 & -99 & 3.30 & 3 & no & -21.32 \\
\hline 1420590 & 150.36694 & 2.616221 & 23.65 & 3.30 & 3.83 & -99 & 3.30 & 3 & no & -21.37 \\
\hline 990122 & 150.29726 & 2.148846 & 20.46 & 2.90 & 3.47 & 3.33 & 3.33 & 3 & yes & -24.62 \\
\hline 1648871 & 150.31337 & 2.716225 & 24.70 & 3.70 & 3.63 & -99 & 3.63 & 3 & no & -20.52 \\
\hline 530538 & 150.27693 & 1.885094 & 24.08 & 4.80 & 4.87 & 4.72 & 4.72 & 3 & no & -22.20 \\
\hline 599355 & 149.85320 & 1.920494 & 22.66 & 3.40 & 0.74 & -99 & 3.40 & 3 & no & -22.17 \\
\hline 1870817 & 150.24617 & 2.858176 & 23.82 & 3.40 & 3.55 & -99 & 3.55 & 3 & no & -21.45 \\
\hline 1454738 & 150.20822 & 2.539911 & 22.46 & 3.30 & 3.24 & -99 & 3.24 & 3 & no & -21.55 \\
\hline
\end{tabular}


Table 3

(Continued)

\begin{tabular}{|c|c|c|c|c|c|c|c|c|c|c|}
\hline ID & $\begin{array}{l}\text { R.A. } \\
\text { (J2000) }\end{array}$ & $\begin{array}{c}\text { Decl. } \\
\text { (J2000) }\end{array}$ & $\begin{array}{c}I_{\mathrm{AB}} \\
(\mathrm{AUTO})\end{array}$ & $z_{\text {est }}$ & $z_{\mathbf{q s o}}$ & $z_{\text {spec }}$ & $z_{\text {used }}$ & Flag & X-Ray & $M_{1450}$ \\
\hline 618000 & 149.83923 & 1.793837 & 24.63 & 3.40 & 3.55 & -99 & 3.55 & 3 & no & -20.77 \\
\hline 1730531 & 149.84322 & 2.659095 & 22.90 & 3.20 & 3.51 & -99 & 3.51 & 3 & no & -22.15 \\
\hline 1970813 & 149.64572 & 2.797057 & 23.24 & 3.40 & 4.15 & -99 & 3.40 & 3 & no & -21.63 \\
\hline 115356 & 150.14893 & 1.587745 & 24.06 & 3.80 & 3.88 & -99 & 3.88 & 3 & no & -21.50 \\
\hline 1717612 & 149.76262 & 2.749090 & 24.81 & 3.90 & 0.49 & -99 & 3.90 & 3 & no & -20.98 \\
\hline 329051 & 150.16891 & 1.774590 & 23.24 & 4.30 & 4.35 & -99 & 4.35 & 3 & no & -22.71 \\
\hline 790476 & 150.11644 & 1.963943 & 23.90 & 3.50 & 3.84 & 3.41 & 3.41 & 3 & no & -21.25 \\
\hline 1326857 & 149.55511 & 2.402630 & 24.32 & 3.30 & 3.94 & -99 & 3.94 & 3 & no & -21.53 \\
\hline 803073 & 150.05769 & 2.046285 & 24.88 & 3.16 & 0.00 & -99 & 3.16 & 3 & no & -21.51 \\
\hline 373838 & 150.02069 & 1.639405 & 24.74 & 3.34 & 3.20 & -99 & 3.20 & 3 & no & -20.43 \\
\hline 374612 & 149.91289 & 1.632472 & 24.07 & 3.12 & 0.06 & -99 & 3.12 & 3 & no & -19.52 \\
\hline 1727644 & 149.85581 & 2.681164 & 24.47 & 3.16 & 0.12 & -99 & 3.16 & 3 & no & -20.71 \\
\hline 376803 & 149.85549 & 1.768740 & 21.06 & 3.30 & 0.09 & -99 & 3.30 & 3 & no & -20.20 \\
\hline 1283260 & 149.78825 & 2.372171 & 23.38 & 3.40 & 0.24 & -99 & 3.40 & 3 & no & -21.61 \\
\hline 594007 & 149.85205 & 1.959338 & 24.39 & 3.26 & 3.35 & -99 & 3.35 & 3 & no & -20.59 \\
\hline 1155791 & 150.66118 & 2.414451 & 24.05 & 3.22 & 3.15 & -99 & 3.15 & 3 & no & -20.97 \\
\hline 1153009 & 150.58006 & 2.439344 & 24.23 & 3.16 & 3.10 & -99 & 3.10 & 3 & no & -20.78 \\
\hline 842269 & 149.58212 & 2.117000 & 23.36 & 3.16 & 0.11 & -99 & 3.16 & 3 & no & -21.62 \\
\hline 851569 & 149.56419 & 2.055025 & 24.68 & 3.50 & 3.75 & -99 & 3.75 & 3 & no & -20.84 \\
\hline 973691 & 150.34480 & 2.257383 & 24.27 & 5.08 & 4.95 & -99 & 4.95 & 3 & no & -21.55 \\
\hline 1200307 & 150.28623 & 2.424521 & 23.34 & 3.10 & 1.18 & -99 & 3.10 & 3 & no & -20.96 \\
\hline 1675730 & 150.07668 & 2.702570 & 23.94 & 3.10 & 0.05 & -99 & 3.10 & 3 & no & -21.07 \\
\hline 1234913 & 150.11537 & 2.363513 & 23.06 & 3.20 & 3.46 & -99 & 3.46 & 3 & no & -21.03 \\
\hline 1413401 & 150.47971 & 2.496383 & 24.24 & 3.12 & 0.00 & -99 & 3.12 & 3 & no & -20.65 \\
\hline 1897688 & 150.07535 & 2.828791 & 23.78 & 3.50 & 0.61 & -99 & 3.50 & 3 & no & -21.27 \\
\hline 502784 & 150.40532 & 1.910871 & 24.09 & 3.12 & 2.62 & -99 & 3.12 & 3 & no & -20.44 \\
\hline 754632 & 150.38487 & 2.036528 & 24.15 & 3.12 & 0.41 & -99 & 3.12 & 3 & no & -20.79 \\
\hline 1464601 & 150.21416 & 2.475017 & 23.19 & 3.11 & 0.81 & -99 & 3.11 & 3 & yes & -21.71 \\
\hline 1498712 & 149.76903 & 2.573805 & 23.41 & 3.14 & 0.24 & -99 & 3.14 & 3 & no & -21.28 \\
\hline 1110682 & 149.50595 & 2.185332 & 22.01 & 3.28 & 0.09 & -99 & 3.28 & 3 & no & -22.71 \\
\hline 1781388 & 149.50613 & 2.642649 & 24.94 & 3.72 & 1.40 & -99 & 3.72 & 3 & no & -20.30 \\
\hline 265707 & 150.70856 & 1.698028 & 23.86 & 3.40 & 0.17 & -99 & 3.40 & 3 & no & -21.03 \\
\hline 593446 & 150.05812 & 1.794438 & 24.44 & 3.20 & 3.16 & -99 & 3.16 & 3 & no & -20.45 \\
\hline 644212 & 149.53085 & 1.953905 & 24.28 & 3.40 & 0.21 & -99 & 3.40 & 3 & no & -20.49 \\
\hline 1043168 & 150.00752 & 2.132265 & 24.25 & 3.40 & 3.33 & -99 & 3.33 & 3 & no & -20.83 \\
\hline 259947 & 150.61942 & 1.737010 & 24.60 & 3.10 & 3.13 & -99 & 3.13 & 3 & no & -20.46 \\
\hline 878986 & 149.47784 & 2.036321 & 24.76 & 4.10 & 4.00 & -99 & 4.00 & 3 & no & -20.61 \\
\hline 262808 & 150.63403 & 1.719672 & 24.96 & 4.60 & 4.46 & -99 & 4.46 & 3 & no & -20.84 \\
\hline 710344 & 150.62828 & 2.006204 & 23.36 & 3.60 & 3.45 & -99 & 3.45 & 3 & no & -22.06 \\
\hline 1384622 & 150.59956 & 2.534162 & 23.29 & 3.20 & 0.53 & -99 & 3.20 & 3 & no & -21.28 \\
\hline 702684 & 150.56259 & 2.060279 & 24.34 & 3.70 & 3.70 & -99 & 3.70 & 3 & no & -20.98 \\
\hline 1255908 & 149.90977 & 2.391198 & 24.69 & 3.90 & 3.85 & -99 & 3.85 & 3 & no & -20.85 \\
\hline 1723659 & 149.77576 & 2.704966 & 17.94 & 3.70 & 3.77 & -99 & 3.77 & 2 & no & -22.76 \\
\hline 428704 & 149.55103 & 1.764459 & 24.59 & 3.10 & 3.20 & -99 & 3.20 & 2 & no & -20.47 \\
\hline 462793 & 150.73788 & 1.884472 & 24.97 & 3.20 & 1.05 & -99 & 3.20 & 2 & no & -19.00 \\
\hline 1041149 & 150.00462 & 2.143163 & 23.88 & 3.22 & 1.04 & -99 & 3.22 & 2 & no & -22.82 \\
\hline 1257886 & 149.97707 & 2.378910 & 24.71 & 3.12 & 0.21 & -99 & 3.12 & 2 & no & -20.50 \\
\hline 1558001 & 149.50140 & 2.469177 & 24.66 & 5.12 & 5.42 & -99 & 5.42 & 2 & no & -22.67 \\
\hline 1309325 & 149.56500 & 2.362180 & 24.65 & 5.60 & 5.28 & -99 & 5.28 & 2 & no & -23.07 \\
\hline 1260818 & 149.92712 & 2.358468 & 24.16 & 3.20 & 3.44 & -99 & 3.44 & 2 & no & -21.06 \\
\hline 405715 & 149.70297 & 1.752171 & 24.95 & 3.10 & 3.38 & -99 & 3.38 & 2 & no & -20.27 \\
\hline 766066 & 150.06465 & 2.124108 & 24.51 & 5.00 & 4.85 & -99 & 4.85 & 2 & no & -21.93 \\
\hline 1631571 & 150.51112 & 2.665648 & 24.61 & 3.00 & 3.82 & -99 & 3.82 & 2 & no & -21.09 \\
\hline 1383442 & 150.67668 & 2.543255 & 23.28 & 3.20 & 0.48 & -99 & 3.20 & 2 & no & -21.09 \\
\hline 361093 & 149.92401 & 1.727810 & 23.83 & 3.33 & 0.67 & -99 & 3.33 & 2 & no & -20.86 \\
\hline 394142 & 149.82733 & 1.665374 & 23.40 & 3.12 & 0.25 & -99 & 3.12 & 2 & no & -21.53 \\
\hline 510917 & 150.49745 & 1.851680 & 24.16 & 4.60 & 4.39 & -99 & 4.39 & 2 & no & -21.82 \\
\hline 380027 & 149.88055 & 1.761200 & 24.01 & 3.60 & 0.63 & -99 & 3.60 & 2 & no & -21.02 \\
\hline 298002 & 150.43706 & 1.649305 & 23.35 & 3.30 & 3.85 & 3.89 & 3.89 & 2 & no & -22.34 \\
\hline 203339 & 149.51596 & 1.609118 & 23.76 & 3.20 & 3.49 & -99 & 3.49 & 2 & no & -20.85 \\
\hline 964908 & 150.42227 & 2.150364 & 23.97 & 3.30 & 0.28 & -99 & 3.30 & 2 & no & -20.89 \\
\hline 1338373 & 149.48837 & 2.327380 & 24.52 & 5.10 & 4.64 & -99 & 4.64 & 2 & no & -21.66 \\
\hline 598191 & 149.80116 & 1.927920 & 24.20 & 4.60 & 4.36 & -99 & 4.36 & 2 & no & -21.75 \\
\hline 232585 & 150.73338 & 1.781481 & 24.11 & 3.20 & 0.22 & -99 & 3.20 & 2 & no & -20.91 \\
\hline 535642 & 150.30261 & 1.852066 & 24.46 & 3.40 & 3.50 & 3.84 & 3.84 & 2 & no & -20.91 \\
\hline
\end{tabular}


Table 3

(Continued)

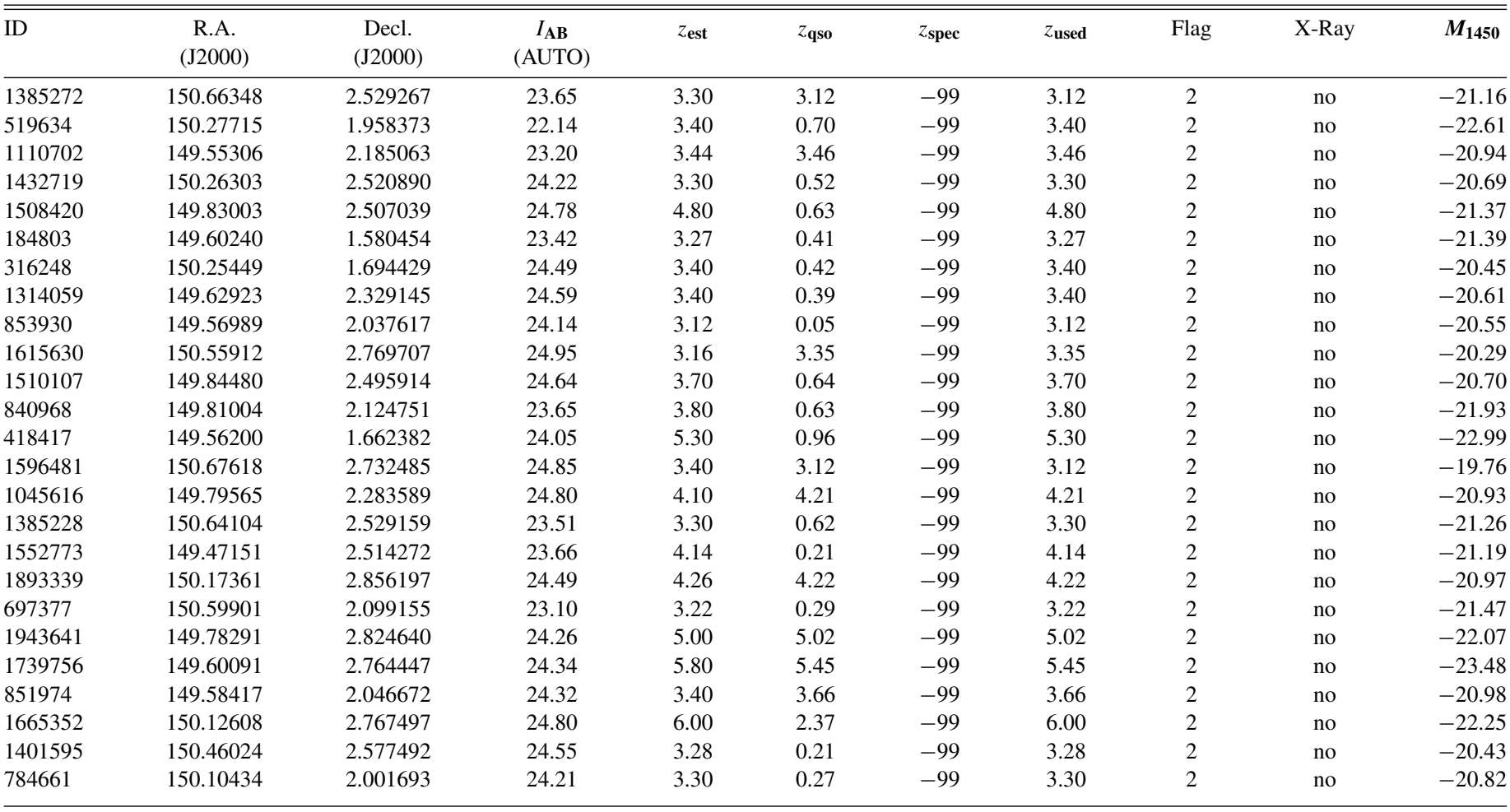

Notes. We give $z_{\text {est }}$, the redshift we estimated based on visual examination, $z_{\mathrm{qso}}$, the photometric redshift from COSMOS, $z_{\text {spec }}$, the spectroscopic redshift, if it exists, and $z_{\text {used }}$, the redshift we adopt in computing the luminosity function. In addition, we list the confidence flag we assigned the source based on visual examination, whether or not the source is detected in X-ray, and the absolute magnitude $M_{1450}$ we derive.

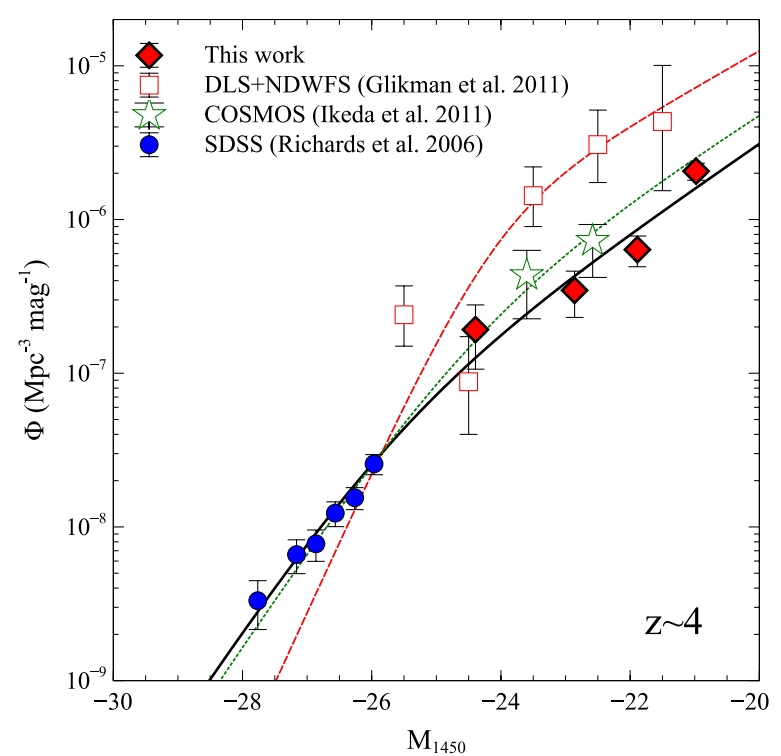

Figure 12. $z \sim 4$ luminosity function derived here compared with previous results. The solid black line is our best fit to the double power-law parameterization including the bright-end result from the SDSS. The dotted green line is the best fit from I11 and the dashed red line is the best fit from G11. The disagreement between the bright-end result of G11 and the SDSS data points arises because G11 recompute the SDSS results after finding the absolute magnitudes directly from the SDSS spectra. Our result is in close agreement with that of III. The disagreement in the faint-end space density between the result derived here and that derived in the DLS+NDWFS is significant, with that result higher by a factor of $\sim 4$.

(A color version of this figure is available in the online journal.) ground-based imaging, which is not as effective as HST imaging at resolving compact, high- $z$ galaxies. Additionally, the number of stellar contaminants in broadband quasar searches grows rapidly at the faintest magnitudes, where spectroscopic followup is the most challenging and thus the contamination is not as well constrained.

In Figure 13, we illustrate the strong evolution of the luminosity function from $z \sim 3.2$ to $z \sim 4$. We find that the space density of faint quasars decreases by a factor of four between $z \sim 3.2$ and $z \sim 4$, in close analogy with the evolution of brighter optical and X-ray quasars at these redshifts. Fontanot et al. (2007) found a relatively low density of faint quasars in the redshift interval $3.5<z<5.2$ from a joint analysis of GOODS and SDSS data, in broad agreement with the results found here. This trend is also verified by very recent results presented in Ikeda et al. (2012, in press), where it is found that the space density of faint quasars at $z \sim 5$ is significantly lower than at $z \sim 4$.

\section{POSSIBLE SOURCES OF ERROR}

\subsection{Cosmic Variance}

The comoving volume probed here at $z \sim 4$ is sufficiently large $\left(2.6 \times 10^{7} \mathrm{Mpc}^{3}\right)$ that we expect cosmic variance to have little effect. However, it has been shown in the SDSS (Shen et al. 2007) that the bias of luminous, high-redshift quasars relative to dark matter halos increases dramatically with redshift, so we attempt to estimate the likely error introduced by cosmic variance. 


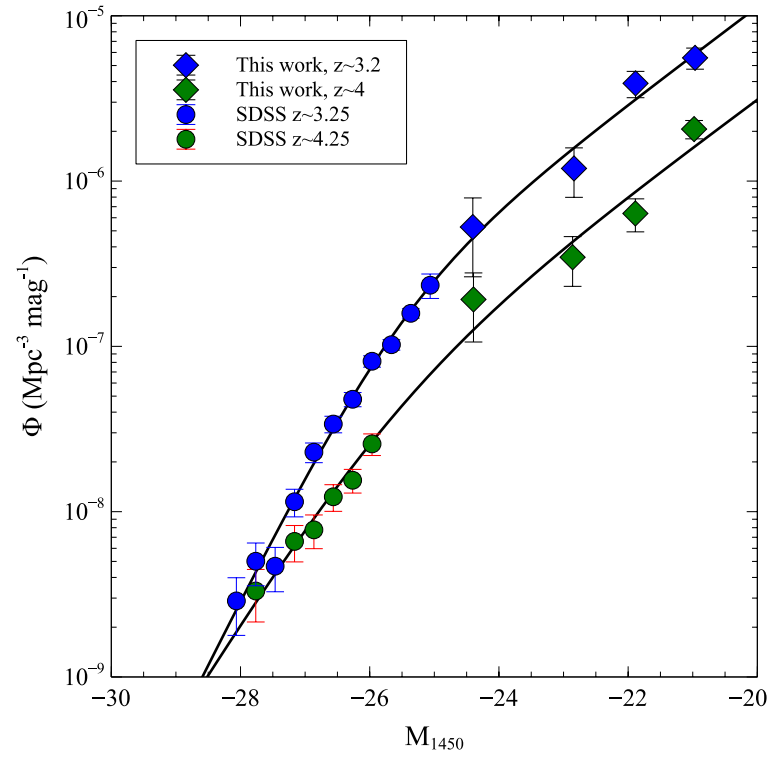

Figure 13. Evolution of the luminosity function from $z \sim 4$ to $z \sim 3.2$, combining the bright end derived from the SDSS (Richards et al. 2006) with the faint end derived here. The space density of faint quasars increases by a factor of $\sim 4$ from $z \sim 4$ to $z \sim 3.2$. Overplotted are the best-fit lines for each luminosity function using the double power-law parameterization. The faint-end slopes we derive for $z \sim 3.2$ and $z \sim 4$ are $\beta=-1.73 \pm 0.11$ and $\beta=-1.72 \pm 0.28$, respectively.

(A color version of this figure is available in the online journal.)

We make use of the cosmic variance cookbook of Moster et al. (2011), in which the bias of galaxies as a function of stellar mass and redshift is used to compute the counting error for galaxies due to cosmic variance. Quasars are expected to be significantly biased with respect to the matter distribution, existing preferentially in the highest mass halos. This leads us to make the approximation that quasars are similarly biased to the most massive $\left(M \geqslant 10^{11} M_{\odot}\right)$ galaxies. The bias factor estimated in this way is in relatively good agreement with the results presented in Shen et al. (2007) for quasar clustering in the SDSS (and may actually be slightly overestimated).

In the redshift window of $3.5<z<5$ in the COSMOS field, we compute an uncertainty of $\sim 19 \%$ in our quasar count, indicating that cosmic variance will contribute only modestly to the derived luminosity function.

\subsection{Contamination by Stars}

The consistent, well-matched wavelength coverage of the COSMOS data, extending from the UV to the infrared, enables the effective removal of stellar contaminants, the SEDs of which differ substantially from those of quasars. Figure 6 illustrates that our final selection preferentially retains objects for which $\chi^{2}$ fitting disfavors stellar templates, and the close agreement with prior studies at $z \sim 3$ also indicates that contamination is low. We note that, even if there were significant stellar contamination in the final sample, this would imply that the luminosity functions are upper bounds, making it more difficult to reconcile the result at $z \sim 4$ found here with that of G11.

\subsection{Contamination by Star-forming Galaxies}

Because we only have spectroscopic confirmation for a fraction of our sample, we may overestimate the faintest end of the luminosity function by inadvertently counting compact, starforming galaxies as quasars. To estimate the contamination from high-redshift galaxies, we analyze a sample of 386 confirmed star-forming galaxies at $z>3$ in COSMOS. These sources were selected as part of a large spectroscopic campaign with Keck DEIMOS (P. Capak et al., in preparation) aimed at finding highredshift galaxies down to $I=25$ from $3<z<6$. A number of techniques (Lyman-break criteria, narrowband selection, etc.) were employed to achieve high completeness, with no selection based on morphology. By applying our quasar selection to these objects, we can get a reasonable sense of the contamination due to compact galaxies.

Of the 386 confirmed high-redshift galaxies, only $2(0.5 \%)$ pass our selection (and were removed from our final sample), with the rest rejected primarily on the basis of morphology. We can combine the fraction of high-redshift star-forming galaxies likely to contaminate our sample with the UV luminosity function for galaxies at $z \sim 4$ (Bouwens et al. 2007) to estimate the level of contamination. Restricting our attention to the faintest absolute magnitude bin $\left(M_{1450}=-21\right)$ examined at $z \sim 4$, we estimate a contamination level of roughly $10^{-6}\left(\mathrm{Mpc}^{-3} \mathrm{mag}^{-1}\right)$ by star-forming galaxies. This is approximately half the space density we derive in this magnitude bin. Therefore, this faintest data point on the luminosity function may be elevated somewhat significantly by star-forming galaxy contamination, leading to an overestimation of the faint-end slope. This issue may need to be settled by follow-up spectroscopy on the faintest sources in the sample.

\subsection{Faint Quasars in Bright Host Galaxies}

Conversely, we may underestimate the faintest bins of the luminosity functions by rejecting bright galaxies hosting faint quasars. This will be the case if a significant fraction of faint type-1 quasars $\left(M_{1450} \gtrsim-23\right)$ exist in galaxies bright enough in the rest-frame UV to make the source appear extended in ACS $I$-band imaging. Two pieces of evidence lead us to believe that this is not the case.

First, we examine 12 Keck DEIMOS spectra of Chandra sources at high redshift that are classified as extended in the ACS catalog, and find that these sources do not show broad lines indicative of a type-1 quasar. Therefore, there is no evidence from spectroscopy of high-redshift X-ray sources of a population of type-1 quasars that we miss by restricting our search to point sources. On the contrary, high-redshift $\mathrm{X}$-ray sources that are morphologically extended seem to be exclusively type-2 AGNs.

Second, we note that, in the sample of $\sim 1400$ high-redshift candidate galaxies in COSMOS followed up on with Keck DEIMOS spectroscopy, we do not find cases of broad-line objects with extended morphology. If a significant number of faint type-1 quasars at $z \sim 4$ have host galaxies bright enough in the rest-frame UV to make the galaxy appear extended in ACS imaging, we should find serendipitous examples of these in a large sample of candidate high-redshift galaxies. In fact we do not; high-redshift, broad-line emitting objects seem invariably to be point sources in the rest-frame UV.

We conclude from these two tests that we do not miss a substantial fraction of type-1 quasars due to our morphological criterion, even at the faintest quasar magnitudes.

\section{COMPARISON OF THE X-RAY AND UV QUASAR LUMINOSITY FUNCTIONS AT HIGH REDSHIFT}

The X-ray luminosity function at $z>3$ in COSMOS was determined recently for the $0.9 \mathrm{deg}^{2}$ Chandra region in 
COSMOS (Civano et al. 2011, hereafter C11). There it is shown that the population of X-ray luminous AGNs decreases rapidly above $z=3$, in agreement with previous results (e.g., Brusa et al. 2009; Silverman et al. 2008). In fact, Figure 4 of C11 shows a decline in space density of X-ray luminous $\left(L_{\mathrm{x}} \gtrsim 10^{44} \mathrm{erg} \mathrm{s}^{-1}\right)$ AGNs by a factor of $\sim 4$ between $z \sim 3.2$ and $z \sim 4$, in agreement with the trend we have demonstrated here for the faint end of the UV QLF at the same redshifts.

A rough sense of the obscured fraction at these redshifts can be obtained simply by comparing the high-redshift X-ray sample with our list of type-1 quasars. Doing so, we find that our selection finds 27/101 sources in the high-redshift X-ray sample. The X-ray sources that we miss are primarily classified as extended in the ACS I-band imaging, or are fainter than $I=25$, or both. As mentioned previously, an examination of 12 spectra of high-redshift X-ray sources that are classified as extended in ACS imaging shows that they are consistent with being type-2 AGNs rather than unobscured quasars. Assuming that the X-ray sources our selection misses are in fact optically obscured, this leads to an estimated type- 2 fraction of roughly $75 \%$.

To more quantitatively compare our results with those found in $\mathrm{C} 11$, we convert our rest-frame UV luminosity functions to $\mathrm{X}$-ray using the $\alpha_{\mathrm{ox}}$ relationship presented in Steffen et al. (2006):

$$
\log \left(l_{2 \mathrm{keV}}\right)=(0.721 \pm 0.011) \log \left(l_{2500 \AA}\right)+(4.531 \pm 0.688) .
$$

We modify this relationship slightly to put it in terms of the luminosity at $1450 \AA$ by assuming a typical quasar spectral slope. With this relationship, the space density of quasars with $L_{\mathrm{x}}>10^{44.15} \mathrm{erg} \mathrm{s}^{-1}$ is found by integrating the UV luminosity functions over the absolute magnitude interval $M_{1450}=$ $[-\infty,-23.45]$. The uncertainty in the conversion is based on the uncertainty in the parameters of the $\alpha_{\text {ox }}$ relation.

Our results are plotted as black diamonds in Figure 14, together with the X-ray results of $\mathrm{C} 11$. The same trend with redshift is observed, but the points derived from the UV QLF are lower by a factor of $\sim 4$, in agreement with the ratio found by directly comparing the two samples. While the $\mathrm{C} 11$ space density is in agreement with the prediction for obscured and unobscured sources (solid line) derived from the Gilli et al. (2007) X-ray background synthesis model, the space density derived from the UV QLF is more consistent with the prediction for unobscured sources only (dashed line). Based on the offset, we again find that the fraction of obscured (but not Comptonthick) quasars is roughly $75 \%$ at $z \sim 3-5$.

It is interesting to compare this result with the redshift evolution of the type- 2 fraction determined by Hasinger (2008, hereafter H08). Figure 9 of that work clearly indicates an upward trend in obscured fraction with redshift, which seems to saturate around $z=2$ at $\sim 60 \%$. This result arises after a correction for the fact that observational bias makes it more difficult to identify obscured AGNs at higher redshifts. The type- 2 fraction we find at $z \sim 3-4, \sim 75 \%$, is higher than the result in H08 and may indicate that the trend of increasing obscured fraction with redshift continues to higher redshifts.

It should also be noted that the result in Figure 9 of H08 is actually a normalization of a rather steep gradient of obscuration versus X-ray luminosity. The obscured fraction decreases with X-ray luminosity at a given redshift, such that for sources above $L_{\mathrm{x}}=10^{44}$ at $z \sim 3-4$ the estimated obscured fraction is actually closer to $20 \%-30 \%$, significantly lower than what

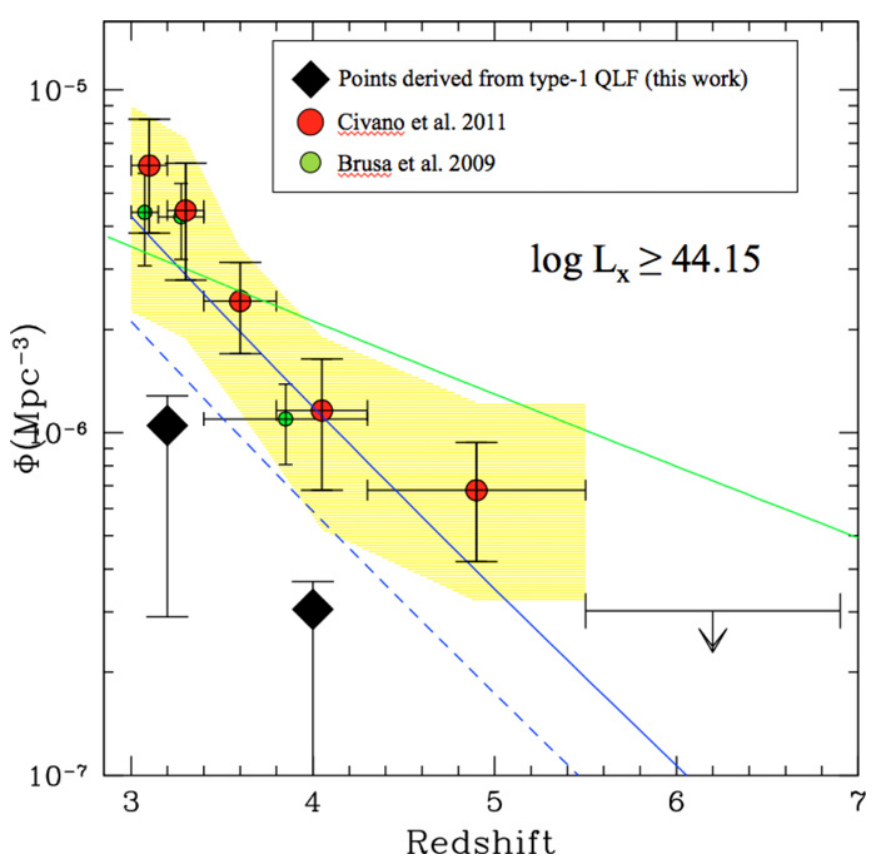

Figure 14. UV luminosity function results, converted to X-ray (black diamonds), compared to the X-ray luminosity functions for X-ray bright Chandra sources derived in Civano et al. (2011) including uncertainties (red circles and yellow shaded area) and for XMM-COSMOS sources (green circles; Brusa et al. 2009). The space density is roughly a fourth of that found in X-ray, implying that $\sim 75 \%$ of bright quasars are optically obscured at these redshifts. The blue lines represent the prediction from the Gilli et al. (2007) model for obscured and unobscured sources (solid) and unobscured sources only (dashed).

(A color version of this figure is available in the online journal.)

we find for comparably luminous sources at these redshifts. Follow-up spectroscopy on more of the $z>3 \mathrm{X}$-ray sources in COSMOS would allow a more firm determination of the type- 2 fraction at these redshifts.

\section{THE IONIZING BACKGROUND DUE TO QUASARS}

Given the luminosity function $\Phi\left(M_{1450}, z\right)$, we can estimate the rate of production of ionizing photons by quasars at redshift $z$. To do so, we use results from Vanden Berk et al. (2001) and Telfer et al. (2002) to approximate the SED of a typical quasar:

$$
L(v) \propto\left\{\begin{array}{ll}
v^{-0.5} & (1216 \AA<\lambda<2500 \AA) \\
v^{-1.76} & (\lambda<1216 \AA)
\end{array} .\right.
$$

The number of hydrogen-ionizing photons emitted per second by a quasar of absolute magnitude $M_{1450}$ is given by

$$
\dot{n}_{\mathrm{HIH}}\left(M_{1450}\right)=\int_{1 \text { ryd }}^{4 \text { ryd }} \frac{L\left(M_{1450}, v\right)}{h v} d v,
$$

where the cutoff at 4 ryd is due to preferential He II absorption of photons above this energy (Madau et al. 1999). Similarly, the rate of emission of He II ionizing photons is given by

$$
\dot{n}_{\mathrm{He} \mathrm{II}}\left(M_{1450}\right)=\int_{4 \text { ryd }}^{\infty} \frac{L\left(M_{1450}, v\right)}{h v} d v .
$$

Combining Equations (5) and (6) with the QLF, one can compute the number of $\mathrm{H}$ I and $\mathrm{He}$ II ionizing photons produced per second per unit comoving volume at a given redshift:

$$
\dot{\mathcal{N}}_{\mathrm{HIH}_{\mathrm{I}}}(z)=\int \Phi(M, z) \dot{n}_{\mathrm{HIH}}(M) d M
$$




$$
\dot{\mathcal{N}}_{\mathrm{He} \text { II }}(z)=\int \Phi(M, z) \dot{n}_{\mathrm{He}}(M) d M .
$$

We use the results for the QLF at $z \sim 3.2$ and $z \sim 4$ presented in Section 7 to estimate these ionizing backgrounds and their evolution with redshift.

\subsection{H I Ionizing Background}

With the QLFs derived in Section 7, we find quasar emission rates of hydrogen-ionizing photons at $z \sim 3.2$ and $z \sim 4$ of $\dot{\mathcal{N}}_{\mathrm{HIH} \text { I }}=3.3 \times 10^{50} \mathrm{~s}^{-1} \mathrm{Mpc}^{-3}$ and $1.0 \times 10^{50} \mathrm{~s}^{-1} \mathrm{Mpc}^{-3}$, respectively. These rates are lower than needed to reionize the IGM at either redshift. At $z=4$, for example, the estimated required rate for reionization is $\dot{\mathcal{N}}_{\mathrm{HIH} \text { I }}=2.4 \times 10^{51} \mathrm{~s}^{-1} \mathrm{Mpc}^{-3}$ (Madau et al. 1999), more than 20 times higher than what we find. In addition, the rates we compute ignore escape fraction and are therefore upper limits on the photons available to ionize the IGM.

The emissivity of UV radiation due to quasars can be found with our luminosity functions and compared with the result given in Haardt \& Madau (2012). We integrate to find the emissivity at $1450 \AA$ and use a conversion factor of $f_{912} \AA / f_{1450 \AA}=$ 0.58 to convert to the corresponding emissivity at $912 \AA$. At $z \sim 3.2$ and $z \sim 4$ we find $\epsilon_{912 \AA}=4.4 \times 10^{24}$ and $1.4 \times$ $10^{24} \mathrm{erg} \mathrm{s}^{-1} \mathrm{Mpc}^{-3} \mathrm{~Hz}^{-1}$, respectively. Haardt \& Madau (2012) find $\epsilon_{912 \AA}=4.3 \times 10^{24}$ and $2.0 \times 10^{24} \mathrm{erg} \mathrm{s}^{-1} \mathrm{Mpc}^{-3} \mathrm{~Hz}^{-1}$ at the same redshifts. We are in relatively close agreement, although our value at $z \sim 4$ is somewhat lower, likely due to the rapid decline in the space density of faint quasars we find.

The emissivity we find at $z \sim 4$ is significantly lower than that required to maintain reionization at $z \sim 7$ (Robertson et al. 2010), even assuming a very high escape fraction for ionizing photons. Therefore, assuming that the faint end of the QLF continues to decline at higher redshifts, type-1 quasars cannot contribute significantly to cosmic reionization of hydrogen at $z \sim 6-10$.

\subsection{He II Ionizing Background}

We find a QSO emission rate of He II-ionizing photons at $z \sim 3.2$ of $\dot{\mathcal{N}}_{\mathrm{He} \text { II }}=3.1 \times 10^{49} \mathrm{~s}^{-1} \mathrm{Mpc}^{-3}$. At $z \sim 4$ we find $\dot{\mathcal{N}}_{\mathrm{He} \text { II }}=1.0 \times 10^{49} \mathrm{~s}^{-1} \mathrm{Mpc}^{-3}$. The significant increase in the production by quasars of He II-ionizing ( $>54.4 \mathrm{eV}$ ) photons from $z \sim 4 \rightarrow 3$ is qualitatively consistent with the observed patchy reionization of He II at $z \sim 3$ (Shull et al. 2010; Bolton et al. 2006). While AGNs are considered the only sources capable of producing sufficient photons with energies above $54.4 \mathrm{eV}$ to be responsible for the reionization of He II, significant uncertainty exists with regard to the specifics of the He II reionization process (Shull et al. 2010). This is mainly due to degeneracies between temperature, density, and ionization fraction in the IGM, as well as the dependence on the spatial distribution and spectral hardness of the ionizing sources. Therefore, we do not attempt to quantify the ionizing background needed to produce the observed He II reionization at $z \sim 3$.

\section{SUMMARY AND CONCLUSIONS}

We have searched the COSMOS field for type-1 quasars at $3.1<z<5$ and $I_{\mathrm{AB}}<25$, with care taken to achieve high completeness. Our method has been to apply a weak quasar selection that is nearly complete but unreliable, and then identify and remove contaminants through careful inspection of the COSMOS photometric and imaging data. This method exploits the fact that stellar contaminants are easily identified with the 29 bands of deep photometry spanning the UV to the infrared. We recover all 39 previously known type-1 quasars at $z>3.1$ in COSMOS as well as 116 additional likely quasars and have presented evidence based on simulations and automated classifiers that this sample is highly complete for type-1 quasars above $z=3.1$ in the HST-ACS region (1.64 deg ${ }^{2}$ ) of COSMOS.

Here we summarize our main conclusions.

1. We find 155 likely type- 1 quasars at $z>3.1$ in the COSMOS field, 39 of which have been previously confirmed, down to a limiting magnitude $I_{\mathrm{AB}}=25$. We present strong evidence that this quasar sample is nearly complete over the redshift range $3.1<z<5$.

2. We use the quasar sample to compute the QLF at $z \sim 3.2$ and $z \sim 4$. The faint-end results we obtain show continuity with the bright-end results reported by Richards et al. (2006) for the SDSS, and demonstrate a clear decrease in the space density of faint quasars, by roughly a factor of four, from $z \sim 3.2$ to $z \sim 4$. The faint end we derive at $z \sim 4$ is in good agreement with the result reported for the COSMOS field in $\mathrm{I} 11$, but disagrees with the result reported in G11. While the source of this discrepancy is unclear, we suggest that it may be caused by contaminating stars/high-redshift galaxies at the faintest magnitudes in the sample of G11.

3. We find no evolution in the faint-end slope $\beta$ over the redshift range investigated. The faint-end slopes we find are $\beta=-1.73 \pm 0.11$ at $z \sim 3.2$ and $\beta=-1.72 \pm 0.28$ at $z \sim 4$, similar to those found at lower redshift.

4. We compare the luminosity functions derived here with the X-ray luminosity function at $z>3$ in COSMOS. The optical QLF and the X-ray QLF evolve similarly between $z \sim 3.2$ and $z \sim 4$ in the sense that both show a rapid decline in space density. However, the different normalizations of the LFs imply that roughly $75 \%$ of AGNs with $\log \left(L_{x}\right) \gtrsim 43.75$ are obscured, type-2 quasars at $z \sim 3-4$.

5. We compute the ionizing background due to quasars, both of $\mathrm{He}$ II and HI. The rapid increase in production of He II ionizing photons from $z \sim 4 \rightarrow 3$ that we find is qualitatively consistent with the observed onset of He II reionization at $z \sim 3$. However, given the decline of the faint end of the QLF to higher redshift, we conclude that faint quasars are unlikely to contribute substantially to cosmic reionization of hydrogen.

We gratefully acknowledge the contributions of the entire COSMOS collaboration. More information about the COSMOS survey is available at http://www.astro.caltech.edu/cosmos. We acknowledge the anonymous referee for suggestions that significantly improved this work. We thank Dr. Eilat Glikman for helpful discussions. M.S. and G.H. acknowledge support by the German Deutsche Forschungsgemeinschaft, DFG Leibniz Prize (FKZ HA 1850/28-1). D.M. gratefully acknowledges the support of the Infrared Processing and Analysis Center (IPAC) Visiting Graduate Fellowship program, as well as the Carnegie Visiting Graduate Fellowship program.

\section{REFERENCES}

Arnouts, S., \& Ilbert, O. 2011, Astrophysics Source Code Library, Record ascl:1108.009, 8009

Bolton, J. S., Haehnelt, M. G., Viel, M., \& Carswell, R. F. 2006, MNRAS, 366,1378 
Bongiorno, A., Zamorani, G., Gavignaud, I., et al. 2007, A\&A, 472, 443 Bouwens, R. J., Illingworth, G. D., Franx, M., \& Ford, H. 2007, ApJ, 670, 928

Boyle, B. J., Shanks, T., \& Peterson, B. A. 1988, MNRAS, 235, 935

Brusa, M., Civano, F., Comastri, A., et al. 2010, ApJ, 716, 348

Brusa, M., Comastri, A., Gilli, R., et al. 2009, ApJ, 693, 8

Capak, P., Aussel, H., Ajiki, M., et al. 2007, ApJS, 172, 99

Civano, F., Brusa, M., Comastri, A., et al. 2011, ApJ, 741, 91

Elvis, M., Civano, F., Vignali, C., et al. 2009, ApJS, 184, 158

Fan, X., Strauss, M. A., Becker, R. H., et al. 2006, AJ, 132, 117

Fontanot, F., Cristiani, S., Monaco, P., et al. 2007, A\&A, 461, 39

Gallagher, S. C., Hines, D. C., Blaylock, M., et al. 2007, ApJ, 665, 157

Gilli, R., Comastri, A., \& Hasinger, G. 2007, A\&A, 463, 79

Glikman, E., Djorgovski, S. G., Stern, D., et al. 2011, ApJ, 728, L26

Haardt, F., \& Madau, P. 2012, ApJ, 746, 125

Hasinger, G. 2008, A\&A, 490, 905

Hasinger, G., Cappelluti, N., Brunner, H., et al. 2007, ApJS, 172, 29

Hopkins, P. F., Somerville, R. S., Hernquist, L., et al. 2006, ApJ, 652, 864

Ikeda, H., Nagao, T., Matsuoka, K., et al. 2011, ApJ, 728, L25

Ikeda, et al. 2012, ApJ, in press (arXiv:1207.1515)

Ilbert, O., Capak, P., Salvato, M., et al. 2009, ApJ, 690, 1236

Jannuzi, B. T., \& Dey, A. 1999, in ASP Conf. Ser. 191, Photometric Redshifts and the Detection of High Redshift Galaxies, ed. R. Weymann, L. StorrieLombardi, M. Sawicki, \& R. Brunner (San Francisco, CA: ASP), 111

Jiang, L., Fan, X., Cool, R. J., et al. 2006, AJ, 131, 2788

Kauffmann, G., \& Haehnelt, M. 2000, MNRAS, 311, 576

Leauthaud, A., Massey, R., Kneib, J.-P., et al. 2007, ApJS, 172, 219

Lilly, S. J., Le Fèvre, O., Renzini, A., et al. 2007, ApJS, 172, 70

Madau, P. 1995, ApJ, 441, 18

Madau, P., Haardt, F., \& Rees, M. J. 1999, ApJ, 514, 648

Marconi, A., Risaliti, G., Gilli, R., et al. 2004, MNRAS, 351, 169

Masters, D., \& Capak, P. 2011, PASP, 123, 638

Moster, B. P., Somerville, R. S., Newman, J. A., \& Rix, H.-W. 2011, ApJ, 731,113
Pickles, A. J. 1998, PASP, 110, 863

Polletta, M., Tajer, M., Maraschi, L., et al. 2007, ApJ, 663, 81

Reimers, D., Kohler, S., Wisotzki, L., et al. 1997, A\&A, 327, 890

Richards, G. T., Myers, A. D., Gray, A. G., et al. 2009, ApJS, 180, 67

Richards, G. T., Strauss, M. A., Fan, X., et al. 2006, AJ, 131, 2766

Richstone, D., Ajhar, E. A., Bender, R., et al. 1998, Nature, 395, A14

Robertson, B. E., Ellis, R. S., Dunlop, J. S., McLure, R. J., \& Stark, D. P. 2010, Nature, 468, 49

Salvato, M., Hasinger, G., Ilbert, O., et al. 2009, ApJ, 690, 1250

Salvato, M., Ilbert, O., Hasinger, G., et al. 2011, ApJ, 742, 61

Sanders, D. B., Salvato, M., Aussel, H., et al. 2007, ApJS, 172, 86

Schmidt, M. 1968, ApJ, 151, 393

Scoville, N., Aussel, H., Brusa, M., et al. 2007, ApJS, 172, 1

Shen, Y., Strauss, M. A., Oguri, M., et al. 2007, AJ, 133, 2222

Shull, J. M., France, K., Danforth, C. W., Smith, B., \& Tumlinson, J. 2010, ApJ, 722,1312

Siana, B., Polletta, M., Smith, H. E., et al. 2008, in ASP Conf. Ser. 381, Infrared Diagnostics of Galaxy Evolution, ed. R.-R. Chary, H. I. Teplitz, \& K. Sheth (San Francisco, CA: ASP), 454

Silverman, J. D., Green, P. J., Barkhouse, W. A., et al. 2008, ApJ, 679, 118

Sokasian, A., Abel, T., \& Hernquist, L. 2002, MNRAS, 332, 601

Steffen, A. T., Strateva, I., Brandt, W. N., et al. 2006, AJ, 131, 2826

Telfer, R. C., Zheng, W., Kriss, G. A., \& Davidsen, A. F. 2002, ApJ, 565, 773

Trump, J. R., Impey, C. D., Elvis, M., et al. 2009, ApJ, 696, 1195

Ueda, Y., Akiyama, M., Ohta, K., \& Miyaji, T. 2003, ApJ, 598, 886

Vanden Berk, D. E., Richards, G. T., Bauer, A., et al. 2001, AJ, 122, 549

Wittman, D. M., Tyson, J. A., Dell'Antonio, I. P., et al. 2002, Proc. SPIE, 4836, 73

Wolf, C., Wisotzki, L., Borch, A., et al. 2003, A\&A, 408, 499

Wyithe, J. S. B., \& Loeb, A. 2003, ApJ, 595, 614

Young, M., Elvis, M., \& Risaliti, G. 2010, ApJ, 708, 1388 